EPJ manuscript No.

(will be inserted by the editor)

\title{
Dimensionally regulated one-loop box scalar integrals with massless internal lines
}

\author{
G. Duplančića and B. Nižić ${ }^{b}$ \\ Theoretical Physics Division, Rudjer Bošković Institute, P.O. Box 180, HR-10002 Zagreb, Croatia \\ Received: date / Revised version: date
}

\begin{abstract}
Using the Feynman parameter method, we have calculated in an elegant manner a set of one-loop box scalar integrals with massless internal lines, but containing 0,1 , 2 , or 3 external massive lines. To treat IR divergences (both soft and collinear), the dimensional regularization method has been employed. The results for these integrals, which appear in the process of evaluating one-loop $(N \geq 5)-$ point integrals and in subdiagrams in QCD loop calculations, have been obtained for arbitrary values of the relevant kinematic variables and presented in a simple and compact form.
\end{abstract}

\section{Introduction}

Scattering processes are one of the most important sources of information on short-distance physics and have played a vital role in establishing the fundamental interactions of nature. In testing various aspects of QCD, the scattering processes in which the total number of particles in the initial and final states is $N \geq 5$ (like $2 \rightarrow 3,2 \rightarrow 4$, etc.) are becoming increasingly important.

The techniques for calculating the tree level amplitudes involving a large number of particles in the final state are well established [1]. Owing to the well-known fact that the LO predictions in perturbative QCD do not have much predictive power, the inclusion of higher-order corrections is essential for many reasons. In general, higherorder corrections have a stabilizing effect reducing the dependence of the LO predictions on the renormalization and factorization scales and the renormalization scheme. Therefore, to achieve a complete confrontation between theoretical predictions and experimental data, it is very important to know the size of radiative corrections to the LO predictions.

Obtaining radiative corrections requires evaluation of one-loop integrals which arise from a Feynman diagramatic approach. The case of massless internal lines is of special interest, because we often deal with either really massless particles (gluons) or particles whose masses can be neglected in high-energy processes (quarks). The main technical difficulty in obtaining the NLO corrections consists in the treatment of the occurring $N$-point tensor and scalar integrals with massless internal lines. In QCD, tensor integrals appear in which the $N$-point integral may contain up to $N$ powers of the loop momentum in the numerator of the integrand. Since these integrals contain

\footnotetext{
a gorand@thphys.irb.hr

b nizic@thphys.irb.hr
}

IR divergences, they need to be calculated in an arbitrary number of dimensions and the standard methods of [2] cannot be directly applied.

Various approaches have been proposed for reducing the dimensionally regulated $(N \geq 5)$-point integrals to a linear combination of $N$ - and lower-point scalar integrals multiplied by tensor structures made from the metric tensor $g^{\mu \nu}$ and external momenta [3, 4, 5, 6, 7].

It has also been shown that the general $(N \geq 5)$-point scalar one-loop integral can be recursively represented as a cyclically symmetric combination of $(N-1)$-point integrals, provided the external momenta are kept in four dimensions [4, 5, 6]. Consequently, all scalar integrals occurring in the computation of an arbitrary one-loop $(N \geq$ $5)$-point integral with massless internal lines can be reduced to a sum over a set of basic scalar box $(N=4)$ integrals with rational coefficients depending on the external momenta and the dimensionality of space-time. This set of diagrams includes IR divergent box integrals with massless internal lines but containing $0,1,2$, and 3 external masses and the IR finite box integral with four external masses.

The IR finite box integral has been evaluated in Ref. [8], and written in a more compact form in Ref. [9]. The results for the IR divergent box integrals with no external masses and with one external mass have been obtained in Refs. 10,11. All IR divergent box integrals have been considered in Ref. [12], using the partial differential equation technique. However, the results obtained for the integrals containing two and three external masses are strictly correct only in the Euclidean region where all relevant kinematical variables are negative.

Being very nontrivial, impossible to check numerically, and of fundamental importance for one-loop calculations in perturbative QCD with massless quarks, it is absolutely essential that these integrals should be evaluated and the 
results of Ref. [12 should be checked using independent techniques.

In this paper we recalculate the IR one-loop box scalar integrals in an elegant manner using dimensional regularisation and the Feynman parameter method, and give results in a simple and compact form. A characteristic feature of our calculation is that the causal i $\epsilon$ has been systematically kept throughout the calculation, so that the results obtained are valid for arbitrary values of the relevant kinematic variables.

The paper is organized as follows. Section 2 is devoted to introducing the notation and to some preliminary considerations. In Sec. 3, using the Feynman parameter method and the dimensional regularization method, we evaluate the IR divergent one-loop box Feynman integrals with massless internal lines but containing $0,1,2$, and 3 massive external lines, and compare our results with the corresponding ones obtained in Ref. 12. Section 4 is devoted to some concluding remarks. An analytical proof of the equivalence of our results to those obtained in Ref. 12] for Euclidean kinematics is given in Appendix A. For the reader's convenience, in Appendix B we present the closed-form expressions for the IR divergent one-loop scalar box integrals evaluated in this paper, i.e., with all poles in $\epsilon_{I R}=D / 2-2$ manifest, and with all functions of the kinematic variables expressed in terms of logarithms and dilogarithms.

\section{Preliminaries}

The massless scalar one-loop box integral in $D$-dimensional space-time is given by

$$
I_{4}\left(p_{1}, p_{2}, p_{3}, p_{4}\right)=\left(\mu^{2}\right)^{2-D / 2} \int \frac{\mathrm{d}^{D} l}{(2 \pi)^{D}} \frac{1}{A_{1} A_{2} A_{3} A_{4}},
$$

where $p_{i}, i=1,2,3,4$ are the external momenta, $l$ is the loop momentum, and $\mu$ is the usual dimensional regularization scale. As indicated in Fig. 1, all external momenta are taken to be incoming, so that the massless propagators have the form

$$
\begin{aligned}
& A_{1}=l^{2}+\mathrm{i} \epsilon, \\
& A_{2}=\left(l+p_{1}\right)^{2}+\mathrm{i} \epsilon, \\
& A_{3}=\left(l+p_{1}+p_{2}\right)^{2}+\mathrm{i} \epsilon, \\
& A_{4}=\left(l+p_{1}+p_{2}+p_{3}\right)^{2}+\mathrm{i} \epsilon .
\end{aligned}
$$

Combining the denominators with the help of the Feynman parametrization formula

$$
\begin{aligned}
& \frac{1}{A_{1} A_{2} A_{3} A_{4}}= \\
& =\int_{0}^{1} \mathrm{~d} x_{1} \mathrm{~d} x_{2} \mathrm{~d} x_{3} \mathrm{~d} x_{4} \frac{3 ! \delta\left(x_{1}+x_{2}+x_{3}+x_{4}-1\right)}{\left(x_{1} A_{1}+x_{2} A_{2}+x_{3} A_{3}+x_{4} A_{4}\right)^{4}}
\end{aligned}
$$

performing the $D$-dimensional loop momentum integration using

$$
\int \frac{\mathrm{d}^{D} l}{(2 \pi)^{D}} \frac{1}{\left(l^{2}-M^{2}+\mathrm{i} \epsilon\right)^{4}}=\frac{\mathrm{i}}{(4 \pi)^{D / 2}} \frac{\Gamma(4-D / 2)}{3 !\left(M^{2}-\mathrm{i} \epsilon\right)^{4-D / 2}},
$$

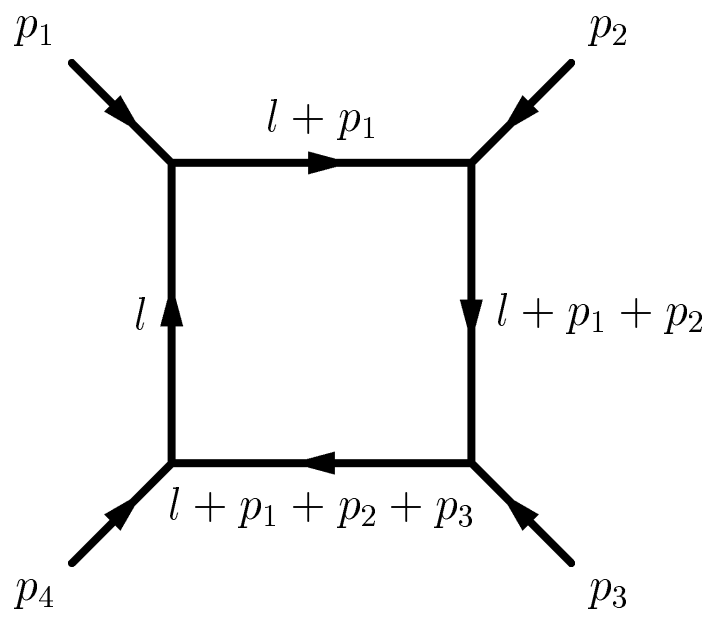

Fig. 1. Basic one-loop box diagram.

introducing the external "masses"

$$
p_{i}^{2}=m_{i}^{2} \quad(i=1,2,3,4),
$$

and the Mandelstam variables

$$
s=\left(p_{1}+p_{2}\right)^{2}, \quad t=\left(p_{2}+p_{3}\right)^{2},
$$

one readily finds that the scalar integral in (11) can be written in the form

$$
\begin{aligned}
& I_{4}\left(s, t ; m_{1}^{2}, m_{2}^{2}, m_{3}^{2}, m_{4}^{2}\right)=\frac{\mathrm{i}}{(4 \pi)^{2}} \frac{\Gamma(4-D / 2)}{\left(4 \pi \mu^{2}\right)^{D / 2-2}} \\
& \quad \times \int_{0}^{1} \mathrm{~d} x_{1} \mathrm{~d} x_{2} \mathrm{~d} x_{3} \mathrm{~d} x_{4} \delta\left(x_{1}+x_{2}+x_{3}+x_{4}-1\right) \\
& \quad \times\left(-x_{1} x_{3} s-x_{2} x_{4} t-x_{1} x_{2} m_{1}^{2}\right. \\
& \left.\quad-x_{2} x_{3} m_{2}^{2}-x_{3} x_{4} m_{3}^{2}-x_{1} x_{4} m_{4}^{2}-\mathrm{i} \epsilon\right)^{D / 2-4} .
\end{aligned}
$$

This is the basic four-point "scalar" parametric integral, serving as a starting point for our further considerations.

Depending on the number of the external massless lines, we distinguish six special cases of this integral. Following the notation of Ref. [12], we denote these integrals by

$$
\begin{aligned}
I_{4}^{4 m} & \equiv I_{4}\left(s, t ; m_{1}^{2}, m_{2}^{2}, m_{3}^{2}, m_{4}^{2}\right), \\
I_{4}^{3 m} & \equiv I_{4}\left(s, t ; 0, m_{2}^{2}, m_{3}^{2}, m_{4}^{2}\right), \\
I_{4}^{2 m h} & \equiv I_{4}\left(s, t ; 0,0, m_{3}^{2}, m_{4}^{2}\right), \\
I_{4}^{2 m e} & \equiv I_{4}\left(s, t ; 0, m_{2}^{2}, 0, m_{4}^{2}\right), \\
I_{4}^{1 m} & \equiv I_{4}\left(s, t ; 0,0,0, m_{4}^{2}\right), \\
I_{4}^{0 m} & \equiv I_{4}(s, t ; 0,0,0,0,),
\end{aligned}
$$

and refer to them as the four-mass scalar box integral, the three-mass box integral, the "hard" two-mass box integral, the "easy" two-mass box integral, the one-mass box integral, and the massless box integral, respectively.

These six box integrals constitute the fundamental set of integrals, in the sense that an arbitrary one-loop $N(\geq$ 
5)-point integral with massless internal lines can be represented in a unique way as a linear combination of these integrals with the coefficients being rational functions of the relevant kinematic variables and the number of space-dimensions $D$.

These integrals arise from the Feynman diagrams depicted in Fig. 2, formally corresponding to the scalar massless $\Phi^{3}$ theory. The thick lines in these diagrams denote the massive (off-shell) external lines. As it is seen from Fig. 2, there are two distinct configurations related to the case when two external lines are massless: the adjacent box diagram $\left(m_{1}^{2}=m_{2}^{2}=0\right)$ and the opposite box diagram $\left(m_{1}^{2}=m_{3}^{2}=0\right)$. They correspond to the hard and easy two-mass box integrals, respectively.

When evaluating the diagrams of Fig. 2, one comes across IR singularities (both collinear and soft). Let us recall the circumstances under which IR singularities appear in a Feynman diagram. When an on-shell quark of momentum $p$ emits a gluon of momentum $k$, then IR singularities appear as a result of vanishing of the quark propagator. If the quark is massless, this can happen when either $p$ and $k$ are collinear ( $k \| p$, collinear singularity) or when the gluon momentum vanishes $(k \rightarrow 0$, soft singularity). Thus, a Feynman diagram with all particles massless will have soft singularity if it contains an internal gluon line attached to two on-shell external quark lines. On the other hand, a diagram will contain collinear singularity if it has an internal gluon line attached to an on-shell external quark line. It follows then that a diagram containing soft singularity contains two collinear singularities at the same time, i.e., soft and collinear singularities overlap.

In view of what has been said above, we conclude that the integral $I_{4}^{4 m}$ is $I R$ finite, and can be calculated in $D=4$ space-time dimensions, while the rest of integrals contain IR divergence $\left(I_{4}^{3 m}\right.$ and $I_{4}^{2 m e}$ collinear divergence, $I_{4}^{2 m h}, I_{4}^{1 m}$, and $I_{4}^{0 m}$ both collinear and soft divergence), and as such have to be evaluated in $D=4+2 \varepsilon_{I R}\left(\varepsilon_{I R}>\right.$ 0) dimensions.

For arbitrary $D$, the integrals (9-13) cannot be expressed by elementary functions, but we know that the expressions corresponding to these diagrams expanded in powers of $\varepsilon_{I R}$ are of the generic form

$$
\sim \frac{A}{\varepsilon_{I R}^{2}}+\frac{B}{\varepsilon_{I R}}+C+\mathcal{O}\left(\epsilon_{I R}\right),
$$

with the coefficients $A, B, C$ being complex functions of the kinematic invariants. The $1 / \varepsilon_{I R}$ poles express the IR divergence.

As stated in the Introduction, the IR divergent integrals $I_{4}^{K}, K \in\{3 m, 2 m h, 2 m e, 1 m, 0 m\}$, defined through Eqs. (7) and (9) - (13) , have been evaluated in Ref. [12] using the partial differential equation technique. After experimenting with various ways to independently derive the results of Ref. [12], we have found that the Feynman parameter method appears to be the most straightforward and satisfactory approach.
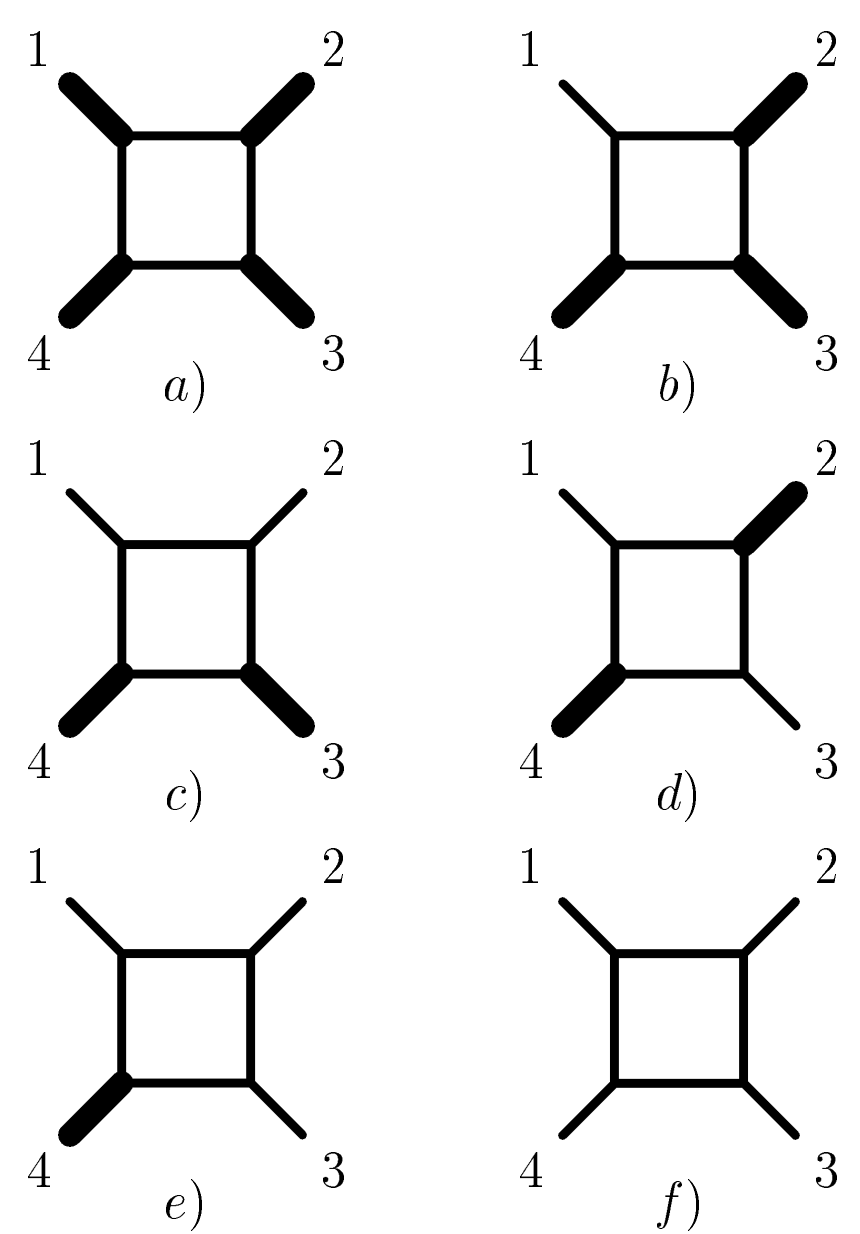

Fig. 2. One-loop box diagrams with massless internal lines but containing $0,1,2,3$, and 4 external massive lines; a) the four-mass box diagram; b) the three-mass box diagram; c) the two-mass box diagram with external masses at adjacent corners (legs 3 and 4); d) the two-mass box diagram with external masses at diagonally opposite corners (legs 2 and 4); e) the one-mass box diagram; f) the massless box diagram. Thick lines designate the massive external lines.

\section{Calculation and results}

Using the Feynman parameter method and the method of dimensional regularization, in this section we evaluate the IR divergent scalar one-loop box integrals $I_{4}^{K}$, $K \in\{3 m, 2 m h, 2 m e, 1 m, 0 m\}$. To accomplish that, we start by considering the most complicated of these integrals, namely, the three-mass box integral $I_{4}^{3 m}$. We show that, paying due attention to the fact that the limit of taking a mass to zero does not necessarily commute with the $\varepsilon_{I R}$ expansion of dimensional regularization, the results obtained at the intermediate steps of the calculation of the integral $I_{4}^{3 m}$ can be used to obtain the results for the rest of the above integrals. A characteristic feature of our calculation is that we keep the causal $\mathrm{i} \epsilon$ systematically through the calculation, so that the results we obtain are valid for arbitrary values of the relevant kinematic variables. 
Let us then start with the three-mass box integral $I_{4}^{3 m}$. By setting $m_{1}^{2}=0$ and $D=4+2 \varepsilon_{I R}\left(\varepsilon_{I R}>0\right)$ in Eq. (7) and eliminating the $\delta$-function by performing the $x_{4}$ integration the integral $I_{4}^{3 m}$ becomes

$$
\begin{aligned}
I_{4}^{3 m}= & \frac{\mathrm{i}}{(4 \pi)^{2}} \frac{\Gamma\left(2-\varepsilon_{I R}\right)}{\left(4 \pi \mu^{2}\right)^{\varepsilon_{I R}}} \int_{0}^{1} \mathrm{~d} x_{1} \int_{0}^{1-x_{1}} \mathrm{~d} x_{2} \int_{0}^{1-x_{1}-x_{2}} \mathrm{~d} x_{3} \\
& \times\left[-x_{1} x_{3} s-x_{2} x_{3} m_{2}^{2}-\left(1-x_{1}-x_{2}-x_{3}\right)\right. \\
& \left.\times\left(x_{1} m_{4}^{2}+x_{2} t+x_{3} m_{3}^{2}\right)-\mathrm{i} \epsilon\right]^{\varepsilon_{I R}-2} .
\end{aligned}
$$

It is a well-known fact that the appropriate choice of Feynman parameters is in practice a critical ingredient in enabling one to evaluate a complicated Feynman integral analytically. There does not appear to be any simple formula for choosing an optimal set of Feynman parameters for a given diagram, as there is generally an enormous set of possibilities.

To proceed with the evaluation of the integral $I_{4}^{3 m}$, the most suitable set of Feynman parameters turns out to be given by 13

$$
\begin{aligned}
& x_{1}=(1-x)(1-y), \\
& x_{2}=x(1-y), \\
& x_{3}=y z
\end{aligned}
$$

The Jacobian corresponding to this transformation of the integration variables is $y(1-y)$. Written in terms of the new variables, the integral (14) takes the form

$$
\begin{aligned}
& I_{4}^{3 m}=\frac{\mathrm{i}}{(4 \pi)^{2}} \frac{\Gamma\left(2-\varepsilon_{I R}\right)}{\left(4 \pi \mu^{2}\right)^{\varepsilon_{I R}}} \int_{0}^{1} \mathrm{~d} x \mathrm{~d} y \mathrm{~d} z y(1-y)\{-y(1-y) \\
& \quad \times\left[(1-x) z s+(1-x)(1-z) m_{4}^{2}+x z m_{2}^{2}+x(1-z) t\right] \\
& \left.\quad-z(1-z) y^{2} m_{3}^{2}-\mathrm{i} \epsilon\right\}^{\varepsilon_{I R}-2} .
\end{aligned}
$$

As it is seen from Eq. (16), the integration over $x$ is elementary and is readily performed. The resulting expression is

$$
\begin{aligned}
I_{4}^{3 m}= & \frac{\kappa}{2} \int_{0}^{1} \mathrm{~d} y \mathrm{~d} z \frac{1}{z\left(s-m_{2}^{2}\right)+(1-z)\left(m_{4}^{2}-t\right)} \\
& \times\left\{\left[-y(1-y)\left(z s+(1-z) m_{4}^{2}\right)\right.\right. \\
& \left.-z(1-z) y^{2} m_{3}^{2}-\mathrm{i} \epsilon\right]^{\varepsilon_{I R}-1} \\
& -\left[-y(1-y)\left(z m_{2}^{2}+(1-z) t\right)\right. \\
& \left.\left.-z(1-z) y^{2} m_{3}^{2}-\mathrm{i} \epsilon\right]^{\varepsilon_{I R}-1}\right\},
\end{aligned}
$$

where we have introduced the abbreviation

$$
\kappa=\frac{\mathrm{i}}{(4 \pi)^{2}} \frac{2 \Gamma\left(1-\varepsilon_{I R}\right)}{\left(4 \pi \mu^{2}\right)^{\varepsilon_{I R}}} .
$$

Next, by pulling out the factor $y^{\varepsilon_{I R}-1}$ from both terms in the curly brackets (which is legitimate since $y$ is positive in the integration region and the sign of $\epsilon(>0)$ does not change), and making use of the following relation:

$$
\begin{aligned}
(a+b-\mathrm{i} \epsilon)^{\varepsilon_{I R}-1}= & \left(\frac{a}{b-\mathrm{i} \epsilon}+1\right)^{\varepsilon_{I R}-1}(b-\mathrm{i} \epsilon)^{\varepsilon_{I R}-1} \\
& (a, b \in \mathbf{R}),
\end{aligned}
$$

(which is not self-evident owing to the fact that $\varepsilon_{I R}$ is not an integer) leads to the integral of the form

$$
\begin{aligned}
& I_{4}^{3 m}=\frac{\kappa}{2} \int_{0}^{1} \mathrm{~d} z \frac{1}{z\left(s-m_{2}^{2}\right)+(1-z)\left(m_{4}^{2}-t\right)} \\
& \quad \times\left\{\left(-z s-(1-z) m_{4}^{2}-\mathrm{i} \epsilon\right)^{\varepsilon_{I R}-1} \int_{0}^{1} \mathrm{~d} y y^{\varepsilon_{I R}-1}\right. \\
& \quad \times\left[1-y\left(1-\frac{z(1-z) m_{3}^{2}+\mathrm{i} \epsilon}{z s+(1-z) m_{4}^{2}+\mathrm{i} \epsilon}\right)\right]^{\varepsilon_{I R}-1} \\
& -\left(-z m_{2}^{2}-(1-z) t-\mathrm{i} \epsilon\right)^{\varepsilon_{I R}-1} \int_{0}^{1} \mathrm{~d} y y^{\varepsilon_{I R}-1} \\
& \left.\quad \times\left[1-y\left(1-\frac{z(1-z) m_{3}^{2}+\mathrm{i} \epsilon}{z m_{2}^{2}+(1-z) t+\mathrm{i} \epsilon}\right)\right]^{\varepsilon_{I R}-1}\right\} .
\end{aligned}
$$

By noticing that the integral over $y$ stands for the Euler integral representation of the hypergeometric function

$$
{ }_{2} F_{1}(a, b ; c ; z)=\frac{\Gamma(c)}{\Gamma(b) \Gamma(c-b)} \int_{0}^{1} \mathrm{~d} t \frac{t^{b-1}(1-t)^{c-b-1}}{(1-t z)^{a}}
$$

$\operatorname{Re} c>\operatorname{Re} b>0 ;|\arg (1-z)|<\pi,(21)$

we obtain the result

$$
\begin{aligned}
& I_{4}^{3 m}=\frac{\kappa}{2 \varepsilon_{I R}} \int_{0}^{1} \mathrm{~d} z \frac{1}{z\left(s-m_{2}^{2}\right)+(1-z)\left(m_{4}^{2}-t\right)} \\
& \quad \times\left[\left(-z s-(1-z) m_{4}^{2}-\mathrm{i} \epsilon\right)^{\varepsilon_{I R}-1}\right. \\
& \quad \times{ }_{2} F_{1}\left(1-\varepsilon_{I R}, \varepsilon_{I R} ; 1+\varepsilon_{I R} ; 1-\frac{z(1-z) m_{3}^{2}+\mathrm{i} \epsilon}{z s+(1-z) m_{4}^{2}+\mathrm{i} \epsilon}\right) \\
& -\left(-z m_{2}^{2}-(1-z) t-\mathrm{i} \epsilon\right)^{\varepsilon_{I R}-1} \\
& \left.\quad \times{ }_{2} F_{1}\left(1-\varepsilon_{I R}, \varepsilon_{I R} ; 1+\varepsilon_{I R} ; 1-\frac{z(1-z) m_{3}^{2}+\mathrm{i} \epsilon}{z m_{2}^{2}+(1-z) t+\mathrm{i} \epsilon}\right)\right] .
\end{aligned}
$$

Next, using the identity (partial fraction decomposition)

$$
\frac{1}{a z+b} \frac{1}{c z+d}=\frac{1}{a d-b c}\left(\frac{a}{a z+b}-\frac{c}{c z+d}\right),
$$

and performing a few simple rearrangements, we find that the integral under consideration can be written in the following form:

$$
I_{4}^{3 m}=\frac{\kappa}{s t-m_{2}^{2} m_{4}^{2}}\left(P^{3 m}+Q^{3 m}\right),
$$


where

$$
\begin{aligned}
& P^{3 m}=\frac{1}{2 \varepsilon_{I R}}\left[\left(m_{2}^{2}-t\right) \int_{0}^{1} \mathrm{~d} z\left[-z m_{2}^{2}-(1-z) t-\mathrm{i} \epsilon\right]^{\varepsilon_{I R}-1}\right. \\
& \times{ }_{2} F_{1}\left(1-\varepsilon_{I R}, \varepsilon_{I R} ; 1+\varepsilon_{I R} ; 1-\frac{z(1-z) m_{3}^{2}+\mathrm{i} \epsilon}{z m_{2}^{2}+(1-z) t+\mathrm{i} \epsilon}\right) \\
& +\left(m_{4}^{2}-s\right) \int_{0}^{1} \mathrm{~d} z\left[-z s-(1-z) m_{4}^{2}-\mathrm{i} \epsilon\right]^{\varepsilon_{I R}-1} \\
& \left.\quad \times{ }_{2} F_{1}\left(1-\varepsilon_{I R}, \varepsilon_{I R} ; 1+\varepsilon_{I R} ; 1-\frac{z(1-z) m_{3}^{2}+\mathrm{i} \epsilon}{z s+(1-z) m_{4}^{2}+\mathrm{i} \epsilon}\right)\right]
\end{aligned}
$$

and

$$
\begin{aligned}
& Q^{3 m}=\frac{1}{2 \varepsilon_{I R}} \int_{0}^{1} \mathrm{~d} z \frac{1}{z-z_{0}} \\
& \times\left[\left(-z m_{2}^{2}-(1-z) t-\mathrm{i} \epsilon\right)^{\varepsilon_{I R}}\right. \\
& \quad \times{ }_{2} F_{1}\left(1-\varepsilon_{I R}, \varepsilon_{I R} ; 1+\varepsilon_{I R} ; 1-\frac{z(1-z) m_{3}^{2}+\mathrm{i} \epsilon}{z m_{2}^{2}+(1-z) t+\mathrm{i} \epsilon}\right) \\
& -\left(-z s-(1-z) m_{4}^{2}-\mathrm{i} \epsilon\right)^{\varepsilon_{I R}} \\
& \left.\quad \times{ }_{2} F_{1}\left(1-\varepsilon_{I R}, \varepsilon_{I R} ; 1+\varepsilon_{I R} ; 1-\frac{z(1-z) m_{3}^{2}+\mathrm{i} \epsilon}{z s+(1-z) m_{4}^{2}+\mathrm{i} \epsilon}\right)\right],
\end{aligned}
$$

with

$$
z_{0}=\frac{t-m_{4}^{2}}{s+t-m_{2}^{2}-m_{4}^{2}} .
$$

In view of (24), it is clear that the integrals $I_{4}^{K}$ can be written in the form

$$
\begin{aligned}
I_{4}^{K}= & \kappa g^{K}\left(P^{K}+Q^{K}\right), \\
& K \in\{3 m, 2 m h, 2 m e, 1 m, 0 m\},
\end{aligned}
$$

with

$$
g^{3 m}=g^{2 m e}=\frac{1}{s t-m_{2}^{2} m_{4}^{2}}, \quad g^{2 m h}=g^{1 m}=g^{0 m}=\frac{1}{s t} .
$$

Not being able to perform the remaining integrations in Eqs. (26) and (25) analytically, we proceed by expanding the integrands in power series in $\varepsilon_{I R}$ and by term by term integration. We shall see below that all divergences of the integral $I_{4}^{K}$ are contained in $P^{K}$, while $Q^{K}$ is completely finite.

It is clear from Eqs. (18) and (24) that to obtain the values of the integrals $I_{4}^{K}, K \in\{3 m, 2 m h, 2 m e, 1 m, 0 m\}$ to order $\mathcal{O}\left(\varepsilon_{I R}^{0}\right)$, the evaluation of the integrals $P^{K}$ and $Q^{K}$ should be made to the same order.

It turns out, however, that the number of integrals that really need to be evaluted reduces to just four, namely, $P^{3 m}$, given by (25), the integrals $P^{2 m h}$ and $P^{2 m e}$, obtained by setting $m_{2}^{2}=0$, and $m_{3}^{2}=0$ in (25), respectively, and the integral $Q^{3 m}$ given by (26). The values of all the other integrals appearing in Eqs. (28) can be derived by taking appropriate zero-mass limits.

\subsection{Calculation of the integrals $P^{3 m}, P^{2 m h}$, and $P^{2 m e}$}

Let us start by considering the integral $P^{3 m}$ given by Eq. (25). Making the change $z \rightarrow 1-z$ in the second term on the right-hand side in (25), one finds that the first and second terms are related to each other by the $t \rightarrow s$ and $m_{2}^{2} \rightarrow m_{4}^{2}$ interchanges. Therefore, the expression for $P^{3 m}$ takes the form

$$
P^{3 m}=\frac{1}{2}\left[R\left(t, m_{2}^{2}, m_{3}^{2}\right)+R\left(s, m_{4}^{2}, m_{3}^{2}\right)\right],
$$

where

$$
\begin{aligned}
& R\left(\alpha, \beta, m_{3}^{2}\right)= \\
& \quad=\frac{1}{\varepsilon_{I R}} \int_{0}^{1} \mathrm{~d} z(\beta-\alpha)(-z \beta-(1-z) \alpha-\mathrm{i} \epsilon)^{\varepsilon_{I R}-1} \\
& \quad \times{ }_{2} F_{1}\left(1-\varepsilon_{I R}, \varepsilon_{I R} ; 1+\varepsilon_{I R} ; 1-\frac{z(1-z) m_{3}^{2}+\mathrm{i} \epsilon}{z \beta+(1-z) \alpha+\mathrm{i} \epsilon}\right) .
\end{aligned}
$$

In order to evaluate this integral, we first apply the linear transformation formula for the hypergeometric functions:

$$
\begin{aligned}
{ }_{2} F_{1}(a, b ; c ; z)= & \frac{\Gamma(c) \Gamma(c-a-b)}{\Gamma(c-a) \Gamma(c-b)} \\
& \times{ }_{2} F_{1}(a, b ; 1+a+b-c ; 1-z) \\
& +\frac{\Gamma(c) \Gamma(a+b-c)}{\Gamma(a) \Gamma(b)}(1-z)^{c-a-b} \\
& \times{ }_{2} F_{1}(c-a, c-b ; 1+c-a-b ; 1-z) \\
& c-a-b \neq \pm n,|\arg (1-z)|<\pi . \quad(32)
\end{aligned}
$$

With Eq. (32) taken into account, (31) becomes

$$
\begin{aligned}
& R\left(\alpha, \beta, m_{3}^{2}\right)= \\
& =\frac{1}{\varepsilon_{I R}} \int_{0}^{1} \mathrm{~d} z(\beta-\alpha)(-z \beta-(1-z) \alpha-\mathrm{i} \epsilon)^{\varepsilon_{I R}-1} \\
& \times\left[\frac{\Gamma\left(1+\varepsilon_{I R}\right) \Gamma\left(\varepsilon_{I R}\right)}{\Gamma\left(2 \varepsilon_{I R}\right)}\right. \\
& \times{ }_{2} F_{1}\left(1-\varepsilon_{I R}, \varepsilon_{I R} ; 1-\varepsilon_{I R} ; \frac{z(1-z) m_{3}^{2}+\mathrm{i} \epsilon}{z \beta+(1-z) \alpha+\mathrm{i} \epsilon}\right) \\
& -\left(\frac{-z(1-z) m_{3}^{2}-\mathrm{i} \epsilon}{-z \beta-(1-z) \alpha-\mathrm{i} \epsilon}\right)^{\varepsilon_{I R}} \\
& \left.\quad \times{ }_{2} F_{1}\left(2 \varepsilon_{I R}, 1 ; 1+\varepsilon_{I R} ; \frac{z(1-z) m_{3}^{2}+\mathrm{i} \epsilon}{z \beta+(1-z) \alpha+\mathrm{i} \epsilon}\right)\right] .
\end{aligned}
$$

Assuming, with no loss of generality, that

$$
\left|m_{3}^{2}\right|<|\alpha|,|\beta| \quad \alpha / \beta>0,
$$

from which it follows

$$
\left|\frac{z(1-z) m_{3}^{2}+\mathrm{i} \epsilon}{z \beta+(1-z) \alpha+\mathrm{i} \epsilon}\right|<1 \quad z \in[0,1]
$$

and making use of the series representation of the hypergeometric function

$$
{ }_{2} F_{1}(a, b ; c ; z)=\sum_{n=0}^{\infty} \frac{\Gamma(a+n) \Gamma(b+n) \Gamma(c)}{\Gamma(a) \Gamma(b) \Gamma(c+n)} \frac{z^{n}}{n !},|z|<1,
$$


leads to the result

$$
\begin{aligned}
& R\left(\alpha, \beta, m_{3}^{2}\right)=\frac{\Gamma\left(\varepsilon_{I R}\right)}{\Gamma\left(2 \varepsilon_{I R}\right)}\left(1-\frac{\beta+\mathrm{i} \epsilon}{\alpha+\mathrm{i} \epsilon}\right) \sum_{n=0}^{\infty}\left(\frac{m_{3}^{2}+\mathrm{i} \epsilon}{\alpha+\mathrm{i} \epsilon}\right)^{n} \\
& \times\left\{(-\alpha-\mathrm{i} \epsilon)^{\varepsilon_{I R}} \frac{\Gamma\left(n+\varepsilon_{I R}\right)}{\Gamma(1+n)}\right. \\
& \times \int_{0}^{1} \mathrm{~d} z[z(1-z)]^{n}\left[1-z\left(1-\frac{\beta+\mathrm{i} \epsilon}{\alpha+\mathrm{i} \epsilon}\right)\right]^{-\left(1+n-\varepsilon_{I R}\right)} \\
& -\left(-m_{3}^{2}-\mathrm{i} \epsilon\right)^{\varepsilon_{I R}} \frac{\Gamma\left(n+2 \varepsilon_{I R}\right)}{\Gamma\left(1+n+\varepsilon_{I R}\right)} \\
& \left.\times \int_{0}^{1} \mathrm{~d} z[z(1-z)]^{n+\varepsilon_{I R}}\left[1-z\left(1-\frac{\beta+\mathrm{i} \epsilon}{\alpha+\mathrm{i} \epsilon}\right)\right]^{-(1+n)}\right\} .
\end{aligned}
$$

Taking into account the integral representation of the hypergeometric function given by Eq. (21) yields

$$
\begin{aligned}
& R\left(\alpha, \beta, m_{3}^{2}\right)=\frac{\Gamma\left(\varepsilon_{I R}\right)}{\Gamma\left(2 \varepsilon_{I R}\right)}\left(1-\frac{\beta+\mathrm{i} \epsilon}{\alpha+\mathrm{i} \epsilon}\right) \sum_{n=0}^{\infty}\left(\frac{m_{3}^{2}+\mathrm{i} \epsilon}{\alpha+\mathrm{i} \epsilon}\right)^{n} \\
& \times\left[(-\alpha-\mathrm{i} \epsilon)^{\varepsilon_{I R}} \frac{\Gamma\left(n+\varepsilon_{I R}\right) \Gamma(1+n)}{\Gamma(2+2 n)}\right. \\
& \times{ }_{2} F_{1}\left(1+n-\varepsilon_{I R}, 1+n ; 2+2 n ; 1-\frac{\beta+\mathrm{i} \epsilon}{\alpha+\mathrm{i} \epsilon}\right) \\
& -\left(-m_{3}^{2}-\mathrm{i} \epsilon\right)^{\varepsilon_{I R}} \frac{\Gamma\left(n+2 \varepsilon_{I R}\right) \Gamma\left(1+n+\varepsilon_{I R}\right)}{\Gamma\left(2+2 n+2 \varepsilon_{I R}\right)} \\
& \left.\times{ }_{2} F_{1}\left(1+n, 1+n+\varepsilon_{I R} ; 2+2 n+2 \varepsilon_{I R} ; 1-\frac{\beta+\mathrm{i} \epsilon}{\alpha+\mathrm{i} \epsilon}\right)\right] .
\end{aligned}
$$

Although obtained under the restriction expressed by Eq. (34), the above expression for $R\left(\alpha, \beta, m_{3}^{2}\right)$ can be analytically continued for an arbitrary value of $\beta$. The series in (38) is convergent because it converges for $\beta=0$ (see below) and the hypergeometric functions reach the maximum at the same point. To retain the convergence of the series, we still assume that $\left|m_{3}^{2}\right|<|\alpha|$.

By inspecting the sum on the right-hand side of (38), we find that, if $\beta \neq 0$, all terms with the exception of the first one $(n=0)$ are of higher order in $\varepsilon_{I R}$ and can therefore be omitted. Upon replacing the whole sum by the first term, and performing a few simple rearrangements, we find

$$
\begin{aligned}
& R\left(\alpha, \beta \neq 0, m_{3}^{2}\right)=\frac{\Gamma^{2}\left(\varepsilon_{I R}\right)}{\Gamma\left(2 \varepsilon_{I R}\right)}\left(1-\frac{\beta+\mathrm{i} \epsilon}{\alpha+\mathrm{i} \epsilon}\right)\left[(-\alpha-\mathrm{i} \epsilon)^{\varepsilon_{I R}}\right. \\
& \times{ }_{2} F_{1}\left(1-\varepsilon_{I R}, 1 ; 2 ; 1-\frac{\beta+\mathrm{i} \epsilon}{\alpha+\mathrm{i} \epsilon}\right) \\
& -\left(-m_{3}^{2}-\mathrm{i} \epsilon\right)^{\varepsilon_{I R}} \frac{1}{2\left(1+2 \varepsilon_{I R}\right)} \\
& \left.\times{ }_{2} F_{1}\left(1,1+\varepsilon_{I R} ; 2+2 \varepsilon_{I R} ; 1-\frac{\beta+\mathrm{i} \epsilon}{\alpha+\mathrm{i} \epsilon}\right)\right]+\mathcal{O}\left(\varepsilon_{I R}\right) .
\end{aligned}
$$

On the basis of formula (21), one can easily show that the first hypergeometric function on the right-hand side of the above expression can be written in the form

$$
\begin{aligned}
& { }_{2} F_{1}\left(1-\varepsilon_{I R}, 1 ; 2 ; 1-\frac{\beta+\mathrm{i} \epsilon}{\alpha+\mathrm{i} \epsilon}\right)= \\
& =\frac{1}{\varepsilon_{I R}}\left(1-\frac{\beta+\mathrm{i} \epsilon}{\alpha+\mathrm{i} \epsilon}\right)^{-1} \frac{(-\alpha-\mathrm{i} \epsilon)^{\varepsilon_{I R}}-(-\beta-\mathrm{i} \epsilon)^{\varepsilon_{I R}}}{(-\alpha-\mathrm{i} \epsilon)^{\varepsilon_{I R}}} .
\end{aligned}
$$

Now, inserting (40) into (39) leads to the expression

$$
\begin{aligned}
& R\left(\alpha, \beta, m_{3}^{2}\right)=\frac{2 \Gamma^{2}\left(\varepsilon_{I R}\right)}{\Gamma\left(1+2 \varepsilon_{I R}\right)}\left[(-\alpha-\mathrm{i} \epsilon)^{\varepsilon_{I R}}-(-\beta-\mathrm{i} \epsilon)^{\varepsilon_{I R}}\right. \\
& -\frac{\left(-m_{3}^{2}-\mathrm{i} \epsilon\right)^{\varepsilon_{I R}}}{2} \frac{\varepsilon_{I R}}{1+2 \varepsilon_{I R}}\left(1-\frac{\beta+\mathrm{i} \epsilon}{\alpha+\mathrm{i} \epsilon}\right) \\
& \left.\times_{2} F_{1}\left(1,1+\varepsilon_{I R} ; 2+2 \varepsilon_{I R} ; 1-\frac{\beta+\mathrm{i} \epsilon}{\alpha+\mathrm{i} \epsilon}\right)\right]+\mathcal{O}\left(\varepsilon_{I R}\right),
\end{aligned}
$$

which is valid for arbitrary values of $\alpha$ and $m_{3}^{2}$, and for $\beta \neq 0$.

On the other hand, if $\beta=0$, the first and second hypergeometric functions appearing on the right-hand side of Eq. (38) reduce to

$$
\frac{\Gamma(2+2 n) \Gamma\left(\varepsilon_{I R}\right)}{\Gamma(1+n) \Gamma\left(1+n+\varepsilon_{I R}\right)}
$$

and

$$
\frac{\Gamma\left(2+2 n+2 \varepsilon_{I R}\right) \Gamma\left(\varepsilon_{I R}\right)}{\Gamma\left(1+n+2 \varepsilon_{I R}\right) \Gamma\left(1+n+\varepsilon_{I R}\right)}
$$

respectively. This implies that when $\beta=0$, all terms in the sum are individually divergent and of the same order in $\varepsilon_{I R}$ and, as such, all have to be taken into account, i. e., the summation has to be performed explicitly. Therefore, as far as the expression for $R\left(\alpha, \beta, m_{3}^{2}\right)$ given by Eq. (38) is concerned, the cases $\beta=0$ and $\beta \neq 0$ ought to be considered separately.

By setting $\beta=0$, Eq. (38) takes the form

$$
\begin{aligned}
& R\left(\alpha, 0, m_{3}^{2}\right)= \\
& \quad=\frac{\Gamma^{2}\left(\varepsilon_{I R}\right)}{\Gamma\left(2 \varepsilon_{I R}\right)}\left[(-\alpha-\mathrm{i} \epsilon)^{\varepsilon_{I R}} \sum_{n=0}^{\infty} \frac{1}{n+\varepsilon_{I R}}\left(\frac{m_{3}^{2}+\mathrm{i} \epsilon}{\alpha+\mathrm{i} \epsilon}\right)^{n}\right. \\
& \left.-\left(-m_{3}^{2}-\mathrm{i} \epsilon\right)^{\varepsilon_{I R}} \sum_{n=0}^{\infty} \frac{1}{n+2 \varepsilon_{I R}}\left(\frac{m_{3}^{2}+\mathrm{i} \epsilon}{\alpha+\mathrm{i} \epsilon}\right)^{n}\right] .
\end{aligned}
$$

For the purpose of performing the summations in (42), we note that the series representation of the hypergeometric function given by (36) particularized for $a=1, c=1+b$ leads to the formula

$$
{ }_{2} F_{1}(1, b ; 1+b ; z)=\sum_{n=0}^{\infty} \frac{b}{n+b} z^{n} \quad|z|<1
$$


which, when taken into account in (42), leads to the result

$$
\begin{aligned}
& R\left(\alpha, 0, m_{3}^{2}\right)=\frac{2 \Gamma^{2}\left(\varepsilon_{I R}\right)}{\Gamma\left(1+2 \varepsilon_{I R}\right)} \\
& \quad \times\left[(-\alpha-\mathrm{i} \epsilon)^{\varepsilon_{I R}}{ }_{2} F_{1}\left(1, \varepsilon_{I R} ; 1+\varepsilon_{I R} ; \frac{m_{3}^{2}+\mathrm{i} \epsilon}{\alpha+\mathrm{i} \epsilon}\right)\right. \\
& \left.\quad-\frac{1}{2}\left(-m_{3}^{2}-\mathrm{i} \epsilon\right)^{\varepsilon_{I R}}{ }_{2} F_{1}\left(1,2 \varepsilon_{I R} ; 1+2 \varepsilon_{I R} ; \frac{m_{3}^{2}+\mathrm{i} \epsilon}{\alpha+\mathrm{i} \epsilon}\right)\right] .
\end{aligned}
$$

This expression for $R\left(\alpha, 0, m_{3}^{2}\right)$ is valid for arbitrary values of $\alpha$ and $m_{3}^{2}$.

To establish contact with the results obtained in Ref. [12], instead of expanding everything in the expressions (41) and (44) to the required order in $\varepsilon_{I R}$, let us, for the time being, expand only the occurring hypergeometric functions. The relevant expansions are

$$
\begin{aligned}
& z_{2} F_{1}(1,1+\delta ; 2+2 \delta ; z)= \\
& \quad=\frac{1+2 \delta}{\delta}\left[1-(1-z)^{\delta}-2 \delta^{2} \operatorname{Li}_{2}(z)+\mathcal{O}\left(\delta^{3}\right)\right]
\end{aligned}
$$

and

$$
{ }_{2} F_{1}(1, \delta ; 1+\delta ; z)=1-\delta \ln (1-z)-\delta^{2} \operatorname{Li}_{2}(z)+\mathcal{O}\left(\delta^{3}\right),
$$

where $\operatorname{Li}_{2}(z)$ stands for the Euler dilogarithm [14] defined as

$$
\operatorname{Li}_{2}(z)=-\int_{0}^{1} \frac{d t}{t} \ln (1-t z) .
$$

Taking (45) into account in (41), we find that

$$
\begin{aligned}
& R\left(\alpha, \beta, m_{3}^{2}\right)=\frac{2 \Gamma^{2}\left(\varepsilon_{I R}\right)}{\Gamma\left(1+2 \varepsilon_{I R}\right)}\left[(-\alpha-\mathrm{i} \epsilon)^{\varepsilon_{I R}}-(-\beta-\mathrm{i} \epsilon)^{\varepsilon_{I R}}\right. \\
& -\frac{1}{2}\left(-m_{3}^{2}-\mathrm{i} \epsilon\right)^{\varepsilon_{I R}}+\frac{1}{2} \frac{(-\beta-\mathrm{i} \epsilon)^{\varepsilon_{I R}}\left(-m_{3}^{2}-\mathrm{i} \epsilon\right)^{\varepsilon_{I R}}}{(-\alpha-\mathrm{i} \epsilon)^{\varepsilon_{I R}}} \\
& \left.+\varepsilon_{I R}^{2} \mathrm{Li}_{2}\left(1-\frac{\beta+\mathrm{i} \epsilon}{\alpha+\mathrm{i} \epsilon}\right)\right]+\mathcal{O}\left(\varepsilon_{I R}\right) .
\end{aligned}
$$

Next, using the expansion (46), the expression (44) becomes

$$
\begin{aligned}
& R\left(\alpha, 0, m_{3}^{2}\right)= \\
& \quad=\frac{2 \Gamma^{2}\left(\varepsilon_{I R}\right)}{\Gamma\left(1+2 \varepsilon_{I R}\right)}\left\{(-\alpha-\mathrm{i} \epsilon)^{\varepsilon_{I R}}-\frac{1}{2}\left(-m_{3}^{2}-\mathrm{i} \epsilon\right)^{\varepsilon_{I R}}\right. \\
& \left.\quad+\varepsilon_{I R}^{2}\left[-\mathrm{Li}_{2}\left(1-\frac{m_{3}^{2}+\mathrm{i} \epsilon}{\alpha+\mathrm{i} \epsilon}\right)+\frac{\pi^{2}}{6}\right]\right\}+\mathcal{O}\left(\varepsilon_{I R}\right)
\end{aligned}
$$

In arriving at (49), the relation

$$
\mathrm{Li}_{2}(1-z)=-\mathrm{Li}_{2}(z)-\ln (z) \ln (1-z)+\frac{\pi^{2}}{6}
$$

has been employed. On the basis of Eqs. (48) and (30) we find that the integral $P^{3 m}$ is given by

$$
P^{3 m}=\frac{\Gamma^{2}\left(\varepsilon_{I R}\right)}{\Gamma\left(1+2 \varepsilon_{I R}\right)}\left\{(-s-\mathrm{i} \epsilon)^{\varepsilon_{I R}}+(-t-\mathrm{i} \epsilon)^{\varepsilon_{I R}}\right.
$$

$$
\begin{aligned}
& -\left(-m_{2}^{2}-\mathrm{i} \epsilon\right)^{\varepsilon_{I R}}-\left(-m_{3}^{2}-\mathrm{i} \epsilon\right)^{\varepsilon_{I R}}-\left(-m_{4}^{2}-\mathrm{i} \epsilon\right)^{\varepsilon_{I R}} \\
& +\frac{1}{2} \frac{\left(-m_{2}^{2}-\mathrm{i} \epsilon\right)^{\varepsilon_{I R}}\left(-m_{3}^{2}-\mathrm{i} \epsilon\right)^{\varepsilon_{I R}}}{(-t-\mathrm{i} \epsilon)^{\varepsilon_{I R}}} \\
& +\frac{1}{2} \frac{\left(-m_{3}^{2}-\mathrm{i} \epsilon\right)^{\varepsilon_{I R}}\left(-m_{4}^{2}-\mathrm{i} \epsilon\right)^{\varepsilon_{I R}}}{(-s-\mathrm{i} \epsilon)^{\varepsilon_{I R}}} \\
& \left.+\varepsilon_{I R}^{2}\left[\operatorname{Li}_{2}\left(1-\frac{m_{2}^{2}+\mathrm{i} \epsilon}{t+\mathrm{i} \epsilon}\right)+\operatorname{Li}_{2}\left(1-\frac{m_{4}^{2}+\mathrm{i} \epsilon}{s+\mathrm{i} \epsilon}\right)\right]\right\} \\
& +\mathcal{O}\left(\varepsilon_{I R}\right) .
\end{aligned}
$$

Similarly, combining Eqs. (48), (49), and (30), we obtain

$$
\begin{aligned}
P^{2 m h}= & \frac{\Gamma^{2}\left(\varepsilon_{I R}\right)}{\Gamma\left(1+2 \varepsilon_{I R}\right)}\left\{(-s-\mathrm{i} \epsilon)^{\varepsilon_{I R}}+(-t-\mathrm{i} \epsilon)^{\varepsilon_{I R}}\right. \\
& -\left(-m_{3}^{2}-\mathrm{i} \epsilon\right)^{\varepsilon_{I R}}-\left(-m_{4}^{2}-\mathrm{i} \epsilon\right)^{\varepsilon_{I R}} \\
& +\frac{1}{2} \frac{\left(-m_{3}^{2}-\mathrm{i} \epsilon\right)^{\varepsilon_{I R}}\left(-m_{4}^{2}-\mathrm{i} \epsilon\right)^{\varepsilon_{I R}}}{(-s-\mathrm{i} \epsilon)^{\varepsilon_{I R}}} \\
& +\varepsilon_{I R}^{2}\left[\frac{\pi^{2}}{6}-\mathrm{Li}_{2}\left(1-\frac{m_{3}^{2}+\mathrm{i} \epsilon}{t+\mathrm{i} \epsilon}\right)\right. \\
& \left.\left.+\operatorname{Li}_{2}\left(1-\frac{m_{4}^{2}+\mathrm{i} \epsilon}{s+\mathrm{i} \epsilon}\right)\right]\right\}+\mathcal{O}\left(\varepsilon_{I R}\right) .
\end{aligned}
$$

We now turn to evaluate the integral $P^{2 m e}$. To this end, we set $m_{3}^{2}=0$ in Eq. (25). As a result, both hypergeometric functions appearing in (25) reduce to

$$
{ }_{2} F_{1}\left(1-\varepsilon_{I R}, \varepsilon_{I R} ; 1+\varepsilon_{I R} ; 1\right)=\frac{\Gamma\left(1+\varepsilon_{I R}\right) \Gamma\left(\varepsilon_{I R}\right)}{\Gamma\left(2 \varepsilon_{I R}\right)},
$$

making it possible to perform the remaining integration analytically. The exact result for the integral $P^{2 m e}$ is

$$
\begin{aligned}
P^{2 m e}= & \frac{\Gamma^{2}\left(\varepsilon_{I R}\right)}{\Gamma\left(1+2 \varepsilon_{I R}\right)}\left[(-s-\mathrm{i} \epsilon)^{\varepsilon_{I R}}+(-t-\mathrm{i} \epsilon)^{\varepsilon_{I R}}\right. \\
& \left.-\left(-m_{2}^{2}-\mathrm{i} \epsilon\right)^{\varepsilon_{I R}}-\left(-m_{4}^{2}-\mathrm{i} \epsilon\right)^{\varepsilon_{I R}}\right] .
\end{aligned}
$$

It is valid for arbitrary values of $m_{2}^{2}$ and $m_{4}^{2}$. Consequently, the expressions for the integrals $P^{1 m}$ and $P^{0 m}$ are obtained by setting $m_{2}^{2}=0$ and $m_{2}^{2}=m_{4}^{2}=0$, respectively, in (54).

A remark concerning the issue of the zero-mass limits of the massive integrals $P^{K}$ is in order. By looking at the expressions for the integrals $P^{K}$ given above, one observes that the limits $P^{2 m h} \rightarrow P^{1 m}, P^{3 m} \rightarrow P^{2 m e}$, and $P^{3 m} \rightarrow P^{2 m h}$ are not smooth. On the other hand, the limits $P^{2 m e} \rightarrow P^{1 m}$ and $P^{1 m} \rightarrow P^{0 m}$ are smooth. In general, there is no reason for the zero- mass limits to be smooth. Namely, the limit of taking a mass to zero does not necessarily commute with the $1 / \varepsilon_{I R}$ expansion of the dimensional regularization, which has been truncated at $\mathcal{O}\left(\varepsilon_{I R}^{0}\right)$. Note, however, that if we were able to evaluate the integral $P^{3 m}$ in 25 ) analytically for general $\varepsilon_{I R}$, the result thus obtained would suffice to obtain the results for the other integrals $P^{2 m h}, P^{2 m e}, P^{1 m}$, and $P^{0 m}$ by simply setting $m_{2}^{2}=0, m_{3}^{2}=0, m_{2}^{2}=m_{3}^{2}=0$, and $m_{2}^{2}=m_{3}^{2}=m_{4}^{2}=0$, respectively, and then expanding these results to the required order in $\varepsilon_{I R}$. 


\subsection{Calculation of the integral $Q^{3 m}$}

As it is seen from (26), the integral $Q^{3 m}$ is given in terms of two hypergeometric functions, both of which can be conveniently written in the form

$$
\begin{gathered}
{ }_{2} F_{1}\left(1-\varepsilon_{I R}, \varepsilon_{I R} ; 1+\varepsilon_{I R} ; 1-\frac{z(1-z) m_{3}^{2}+\mathrm{i} \epsilon}{z \alpha+(1-z) \beta+\mathrm{i} \epsilon}\right) \\
(\alpha, \beta) \in\left\{\left(m_{2}^{2}, t\right),\left(s, m_{4}^{2}\right)\right\} .
\end{gathered}
$$

Making use of the transformation formula

$$
{ }_{2} F_{1}(a, b ; c ; z)=(1-z)^{c-a-b}{ }_{2} F_{1}(c-a, c-b ; c ; z),
$$

the symmetry of ${ }_{2} F_{1}$ with respect to the arguments $a$ and $b$, i.e.,

$$
{ }_{2} F_{1}(a, b ; c ; z)={ }_{2} F_{1}(b, a ; c ; z),
$$

and the integral representation of the hypergeometric function (21), we can write the hypergeometric function given by (55) in the form

$$
\begin{aligned}
& { }_{2} F_{1}\left(1-\varepsilon_{I R}, \varepsilon_{I R} ; 1+\varepsilon_{I R} ; 1-\frac{z(1-z) m_{3}^{2}+\mathrm{i} \epsilon}{z \alpha+(1-z) \beta+\mathrm{i} \epsilon}\right)= \\
& =\left(\frac{-z(1-z) m_{3}^{2}-\mathrm{i} \epsilon}{-z \alpha-(1-z) \beta-\mathrm{i} \epsilon}\right)^{\varepsilon_{I R}} \frac{\Gamma\left(1+\varepsilon_{I R}\right)}{\Gamma\left(1-\varepsilon_{I R}\right) \Gamma\left(2 \varepsilon_{I R}\right)} \\
& \times \int_{0}^{1} \mathrm{~d} y y^{2 \varepsilon_{I R}-1}(1-y)^{-\varepsilon_{I R}} \\
& \times\left[1-y\left(1-\frac{z(1-z) m_{3}^{2}+\mathrm{i} \epsilon}{z \alpha+(1-z) \beta+\mathrm{i} \epsilon}\right)\right]^{-1} .
\end{aligned}
$$

Upon substituting (58) into (26), and utilizing the identity (23) once more, we find that

$$
\begin{aligned}
& Q^{3 m}=\frac{\Gamma\left(\varepsilon_{I R}\right)}{2 \Gamma\left(1-\varepsilon_{I R}\right) \Gamma\left(2 \varepsilon_{I R}\right)} \int_{0}^{1} \mathrm{~d} z \frac{1}{z-z_{0}} \\
& \quad \times\left[-z(1-z) m_{3}^{2}-\mathrm{i} \epsilon\right]^{\varepsilon_{I R}} \int_{0}^{1} \mathrm{~d} y y^{2 \varepsilon_{I R}}(1-y)^{-\varepsilon_{I R}} \\
& \quad \times\left\{\left[-z(1-z) m_{3}^{2}+z s+(1-z) m_{4}^{2}\right]\right. \\
& \quad \times\left[-z s-(1-z) m_{4}^{2}-\mathrm{i} \epsilon\right. \\
& \left.\quad+y\left(-z(1-z) m_{3}^{2}+z s+(1-z) m_{4}^{2}\right)\right]^{-1} \\
& \quad-\left[-z(1-z) m_{3}^{2}+z m_{2}^{2}+(1-z) t\right] \\
& \quad \times\left[-z m_{2}^{2}-(1-z) t-\mathrm{i} \epsilon\right. \\
& \left.\left.\quad+y\left(-z(1-z) m_{3}^{2}+z m_{2}^{2}+(1-z) t\right)\right]^{-1}\right\}
\end{aligned}
$$

Since we are interested in obtaining the value of $Q^{3 m}$ to $\mathcal{O}\left(\varepsilon_{I R}^{0}\right)$, the fact that the expansion of the prefactor in the above expression is of the form $1+\mathcal{O}\left(\varepsilon_{I R}\right)$, allows us to set $\varepsilon_{I R}=0$ in the integrand in (59). As a result, the expression for $Q^{3 m}$ reduces to

$$
\begin{aligned}
& Q^{3 m}=\int_{0}^{1} \mathrm{~d} z \frac{1}{z-z_{0}} \int_{0}^{1} \mathrm{~d} y \\
& \quad \times\left\{\left[-z(1-z) m_{3}^{2}+z s+(1-z) m_{4}^{2}\right]\right.
\end{aligned}
$$

$$
\begin{aligned}
& \times\left[-z s-(1-z) m_{4}^{2}-\mathrm{i} \epsilon\right. \\
& \left.+y\left(-z(1-z) m_{3}^{2}+z s+(1-z) m_{4}^{2}\right)\right]^{-1} \\
& -\left[-z(1-z) m_{3}^{2}+z m_{2}^{2}+(1-z) t\right] \\
& \times\left[-z m_{2}^{2}-(1-z) t-\mathrm{i} \epsilon\right. \\
& \left.\left.+y\left(-z(1-z) m_{3}^{2}+z m_{2}^{2}+(1-z) t\right)\right]^{-1}\right\}+\mathcal{O}\left(\varepsilon_{I R}\right) .
\end{aligned}
$$

Performing the $y$ integration, we find

$$
Q^{3 m}=\int_{0}^{1} \frac{\mathrm{d} z}{z-z_{0}} \ln \left(\frac{z\left(t-m_{2}^{2}\right)-t-\mathrm{i} \epsilon}{z\left(m_{4}^{2}-s\right)-m_{4}^{2}-\mathrm{i} \epsilon}\right)+\mathcal{O}\left(\varepsilon_{I R}\right) .
$$

To carry out the remaining integration, it is important to note that the residue of the integrand at the pole $z_{0}$ is zero, and the logarithm does not cross the cut. This fact allows us to make a few simple transformations of the integrand.

Thus, upon the substitution $z \rightarrow z+z_{0}$, the decomposition

$$
\int_{0}^{1} d z \rightarrow-\int_{0}^{-z_{0}} d z+\int_{0}^{1-z_{0}} d z
$$

followed by a change of the variable $z \rightarrow-z z_{0}$ in the first, and $z \rightarrow z\left(1-z_{0}\right)$ in the second term, the integral $Q^{3 m}$ can be written down as

$$
\begin{aligned}
Q^{3 m}= & \int_{0}^{1} \frac{\mathrm{d} z}{z}\left\{\ln \left[1-z\left(1-\left(m_{2}^{2}+\mathrm{i} \epsilon\right) f^{3 m}\right)\right]\right. \\
& +\ln \left[1-z\left(1-\left(m_{4}^{2}+\mathrm{i} \epsilon\right) f^{3 m}\right)\right]- \\
& -\ln \left[1-z\left(1-(s+\mathrm{i} \epsilon) f^{3 m}\right)\right] \\
& \left.-\ln \left[1-z\left(1-(t+\mathrm{i} \epsilon) f^{3 m}\right)\right]\right\}+\mathcal{O}\left(\varepsilon_{I R}\right)
\end{aligned}
$$

where we have introduced the abbreviation

$$
f^{3 m}=\frac{s+t-m_{2}^{2}-m_{4}^{2}}{s t-m_{2}^{2} m_{4}^{2}} .
$$

Expressed in terms of the Euler dilogarithm, the final result for the integral $Q^{3 m}$ takes the form

$$
\begin{aligned}
& Q^{3 m}=\mathrm{Li}_{2}\left[1-(s+\mathrm{i} \epsilon) f^{3 m}\right]+\operatorname{Li}_{2}\left[1-(t+\mathrm{i} \epsilon) f^{3 m}\right] \\
& -\mathrm{Li}_{2}\left[1-\left(m_{2}^{2}+\mathrm{i} \epsilon\right) f^{3 m}\right]-\mathrm{Li}_{2}\left[1-\left(m_{4}^{2}+\mathrm{i} \epsilon\right) f^{3 m}\right]+\mathcal{O}\left(\varepsilon_{I R}\right) .
\end{aligned}
$$

The expression for $Q^{3 m}$ does not depend on $m_{3}^{2}$, and is valid for arbitrary values of $m_{2}^{2}$ and $m_{4}^{2}$. A consequence of this is that the expression for the integral $Q^{2 m h}$ to the same order in $\varepsilon_{I R}$ can simply be obtained by setting $m_{2}^{2}=0$ in (64). Note, however, that, strictly speaking, the expressions for $Q^{2 m e}, Q^{1 m}$, and $Q^{0 m}$ cannot be obtained by taking appropriate zero-mass limits of the same expression. Namely, the expression (59) has been derived assuming that $m_{3}^{2} \neq 0$. Therefore, the $\varepsilon_{I R}$ expansion of (59) is not justified in the $m_{3}^{2} \rightarrow 0$ limit. In order to obtain the integrals $Q^{2 m e}, Q^{1 m}$, and $Q^{0 m}$, we proceed as follows. We return to Eq. (26) and set $m_{3}^{2}=0$. As a result, Eq. 
(26) reduces to

$$
\begin{aligned}
Q^{2 m e}= & \frac{\Gamma^{2}\left(\varepsilon_{I R}\right)}{2 \Gamma\left(2 \varepsilon_{I R}\right)} \int_{0}^{1} \mathrm{~d} z \frac{1}{z-z_{0}}\left[\left(-z m_{2}^{2}-(1-z) t-\mathrm{i} \epsilon\right)^{\varepsilon_{I R}}\right. \\
& \left.-\left(-z s-(1-z) m_{4}^{2}-\mathrm{i} \epsilon\right)^{\varepsilon_{I R}}\right] .
\end{aligned}
$$

Expanding this expression into power series in $\varepsilon_{I R}$

$$
Q^{2 m e}=\int_{0}^{1} \frac{\mathrm{d} z}{z-z_{0}} \ln \left(\frac{z\left(t-m_{2}^{2}\right)-t-\mathrm{i} \epsilon}{z\left(m_{4}^{2}-s\right)-m_{4}^{2}-\mathrm{i} \epsilon}\right)+\mathcal{O}\left(\varepsilon_{I R}\right),
$$

and comparing it with the expansion of $Q^{3 m}$ given by Eq. 61), we find that the expansions for $Q^{3 m}$ and $Q^{2 m e}$ coincide to the required order in $\varepsilon_{I R}$. This being the case, all other intgerals $Q^{K}$ can be derived from the integral $Q^{3 m}$ given by Eq. (64) by taking appropriate zero-mass limits.

\subsection{Results}

Having obtained, in the preceding subsections, the closed form expressions for the integrals $P^{K}\left(s, t ;\left\{m_{i}^{2}\right\}\right)$ and $Q^{K}\left(s, t ;\left\{m_{i}^{2}\right\}\right)$ to order $\mathcal{O}\left(\varepsilon_{I R}^{0}\right)$, we are now in a position to write down explicit expressions for the integrals $I_{4}^{K}\left(s, t ;\left\{m_{i}^{2}\right\}\right)$.

Before proceeding, it is convenient to introduce the functions

$$
\begin{aligned}
f^{3 m}=f^{2 m e} & =\frac{s+t-m_{2}^{2}-m_{4}^{2}}{s t-m_{2}^{2} m_{4}^{2}}, \\
f^{2 m h}=f^{1 m} & =\frac{s+t-m_{4}^{2}}{s t}, \\
f^{0 m} & =\frac{s+t}{s t},
\end{aligned}
$$

and the notation

$$
r_{\Gamma}=\frac{\Gamma\left(1-\varepsilon_{I R}\right) \Gamma^{2}\left(1+\varepsilon_{I R}\right)}{\Gamma\left(1+2 \varepsilon_{I R}\right)}
$$

for the $\Gamma$ function prefactor.

Now, on the basis of Eqs. 28, (64), (51), (52), and (54), we find that

the three-mass scalar box integral is

$$
\begin{aligned}
& I_{4}^{3 m}\left(s, t ; m_{2}^{2}, m_{3}^{2}, m_{4}^{2}\right)=\frac{\mathrm{i}}{(4 \pi)^{2}} \frac{r_{\Gamma}}{s t-m_{2}^{2} m_{4}^{2}} \\
& \quad \times\left\{\frac { 2 } { \varepsilon _ { I R } ^ { 2 } } \left[\left(\frac{-s-\mathrm{i} \epsilon}{4 \pi \mu^{2}}\right)^{\varepsilon_{I R}}+\left(\frac{-t-\mathrm{i} \epsilon}{4 \pi \mu^{2}}\right)^{\varepsilon_{I R}}-\left(\frac{-m_{2}^{2}-\mathrm{i} \epsilon}{4 \pi \mu^{2}}\right)^{\varepsilon_{I R}}\right.\right. \\
& \left.\quad-\left(\frac{-m_{3}^{2}-\mathrm{i} \epsilon}{4 \pi \mu^{2}}\right)^{\varepsilon_{I R}}-\left(\frac{-m_{4}^{2}-i \epsilon}{4 \pi \mu^{2}}\right)^{\varepsilon_{I R}}\right] \\
& +\frac{1}{\varepsilon_{I R}^{2}}\left(\frac{-m_{2}^{2}-\mathrm{i} \epsilon}{4 \pi \mu^{2}}\right)^{\varepsilon_{I R}}\left(\frac{-m_{3}^{2}-\mathrm{i} \epsilon}{4 \pi \mu^{2}}\right)^{\varepsilon_{I R}}\left(\frac{-t-\mathrm{i} \epsilon}{4 \pi \mu^{2}}\right)^{-\varepsilon_{I R}} \\
& +\frac{1}{\varepsilon_{I R}^{2}}\left(\frac{-m_{3}^{2}-\mathrm{i} \epsilon}{4 \pi \mu^{2}}\right)^{\varepsilon_{I R}}\left(\frac{-m_{4}^{2}-\mathrm{i} \epsilon}{4 \pi \mu^{2}}\right)^{\varepsilon_{I R}}\left(\frac{-s-\mathrm{i} \epsilon}{4 \pi \mu^{2}}\right)^{-\varepsilon_{I R}}
\end{aligned}
$$

$$
\begin{aligned}
& +2 \operatorname{Li}_{2}\left(1-\frac{m_{2}^{2}+\mathrm{i} \epsilon}{t+\mathrm{i} \epsilon}\right)+2 \operatorname{Li}_{2}\left(1-\frac{m_{4}^{2}+\mathrm{i} \epsilon}{s+\mathrm{i} \epsilon}\right) \\
& +2 \operatorname{Li}_{2}\left[1-(s+\mathrm{i} \epsilon) f^{3 m}\right]+2 \operatorname{Li}_{2}\left[1-(t+\mathrm{i} \epsilon) f^{3 m}\right] \\
& -2 \operatorname{Li}_{2}\left[1-\left(m_{2}^{2}+\mathrm{i} \epsilon\right) f^{3 m}\right] \\
& \left.-2 \operatorname{Li}_{2}\left[1-\left(m_{4}^{2}+\mathrm{i} \epsilon\right) f^{3 m}\right]\right\}+\mathcal{O}\left(\epsilon_{I R}\right),
\end{aligned}
$$

the adjacent ("hard") two-mass scalar box integral is

$$
\begin{aligned}
& I_{4}^{2 m h}\left(s, t ; m_{3}^{2}, m_{4}^{2}\right)=\frac{\mathrm{i}}{(4 \pi)^{2}} \frac{r_{\Gamma}}{s t}\left\{\frac { 2 } { \varepsilon _ { I R } ^ { 2 } } \left[\left(\frac{-s-\mathrm{i} \epsilon}{4 \pi \mu^{2}}\right)^{\varepsilon_{I R}}\right.\right. \\
& \left.+\left(\frac{-t-\mathrm{i} \epsilon}{4 \pi \mu^{2}}\right)^{\varepsilon_{I R}}-\left(\frac{-m_{3}^{2}-\mathrm{i} \epsilon}{4 \pi \mu^{2}}\right)^{\varepsilon_{I R}}-\left(\frac{-m_{4}^{2}-\mathrm{i} \epsilon}{4 \pi \mu^{2}}\right)^{\varepsilon_{I R}}\right] \\
& +\frac{1}{\varepsilon_{I R}^{2}}\left(\frac{-m_{3}^{2}-\mathrm{i} \epsilon}{4 \pi \mu^{2}}\right)^{\varepsilon_{I R}}\left(\frac{-m_{4}^{2}-\mathrm{i} \epsilon}{4 \pi \mu^{2}}\right)^{\varepsilon_{I R}}\left(\frac{-s-\mathrm{i} \epsilon}{4 \pi \mu^{2}}\right)^{-\varepsilon_{I R}} \\
& +2 \operatorname{Li}_{2}\left(1-\frac{m_{4}^{2}+\mathrm{i} \epsilon}{s+\mathrm{i} \epsilon}\right)-2 \operatorname{Li}_{2}\left(1-\frac{m_{3}^{2}+\mathrm{i} \epsilon}{t+\mathrm{i} \epsilon}\right) \\
& +2 \operatorname{Li}_{2}\left[1-(s+\mathrm{i} \epsilon) f^{2 m h}\right]+2 \operatorname{Li}_{2}\left[1-(t+\mathrm{i} \epsilon) f^{2 m h}\right] \\
& \left.-2 \operatorname{Li}_{2}\left[1-\left(m_{4}^{2}+\mathrm{i} \epsilon\right) f^{2 m h}\right]\right\}+\mathcal{O}\left(\epsilon_{I R}\right),
\end{aligned}
$$

the opposite ("easy") two-mass scalar box integral is

$$
\begin{aligned}
& I_{4}^{2 m e}\left(s, t ; m_{2}^{2}, m_{4}^{2}\right)=\frac{\mathrm{i}}{(4 \pi)^{2}} \frac{r_{\Gamma}}{s t-m_{2}^{2} m_{4}^{2}} \\
& \left\{\frac { 2 } { \varepsilon _ { I R } ^ { 2 } } \left[\left(\frac{-s-\mathrm{i} \epsilon}{4 \pi \mu^{2}}\right)^{\varepsilon_{I R}}+\left(\frac{-t-\mathrm{i} \epsilon}{4 \pi \mu^{2}}\right)^{\varepsilon_{I R}}-\left(\frac{-m_{2}^{2}-\mathrm{i} \epsilon}{4 \pi \mu^{2}}\right)^{\varepsilon_{I R}}\right.\right. \\
& \left.-\left(\frac{-m_{4}^{2}-\mathrm{i} \epsilon}{4 \pi \mu^{2}}\right)^{\varepsilon_{I R}}\right]+2 \operatorname{Li}_{2}\left[1-(s+\mathrm{i} \epsilon) f^{2 m e}\right] \\
& +2 \operatorname{Li}_{2}\left[1-(t+\mathrm{i} \epsilon) f^{2 m e}\right]-2 \operatorname{Li}_{2}\left[1-\left(m_{2}^{2}+\mathrm{i} \epsilon\right) f^{2 m e}\right] \\
& \left.-2 \operatorname{Li}_{2}\left[1-\left(m_{4}^{2}+\mathrm{i} \epsilon\right) f^{2 m e}\right]\right\}+\mathcal{O}\left(\epsilon_{I R}\right) .
\end{aligned}
$$

An essential feature of the above expression for $I_{4}^{2 m e}$ is that it is well behaved in the $m_{2}^{2} \rightarrow 0, m_{4}^{2} \rightarrow 0$ limits. A consequence of this is that it contains both the one-mass scalar box integral and the massless scalar box integral. Thus, setting $m_{2}^{2}=0$ in (71) and making use of the fact that $\operatorname{Li}_{2}(1)=\pi^{2} / 6$, we find that the one-mass box scalar integral is

$$
\begin{aligned}
& I_{4}^{1 m}\left(s, t ; m_{4}^{2}\right)=\frac{\mathrm{i}}{(4 \pi)^{2}} \frac{r_{\Gamma}}{s t}\left\{\frac { 2 } { \varepsilon _ { I R } ^ { 2 } } \left[\left(\frac{-s-\mathrm{i} \epsilon}{4 \pi \mu^{2}}\right)^{\varepsilon_{I R}}\right.\right. \\
& \left.+\left(\frac{-t-\mathrm{i} \epsilon}{4 \pi \mu^{2}}\right)^{\varepsilon_{I R}}-\left(\frac{-m_{4}^{2}-\mathrm{i} \epsilon}{4 \pi \mu^{2}}\right)^{\varepsilon_{I R}}\right] \\
& +2 \mathrm{Li}_{2}\left[1-(s+\mathrm{i} \epsilon) f^{1 m}\right]+2 \mathrm{Li}_{2}\left[1-(t+\mathrm{i} \epsilon) f^{1 m}\right] \\
& \left.-2 \operatorname{Li}_{2}\left[1-\left(m_{4}^{2}+\mathrm{i} \epsilon\right) f^{1 m}\right]-\frac{\pi^{2}}{3}\right\}+\mathcal{O}\left(\epsilon_{I R}\right) .
\end{aligned}
$$


Finally, setting $m_{4}^{2}=0$ in (72), we find that the massless scalar box integral is given by

$$
\begin{aligned}
& I_{4}^{0 m}(s, t)=\frac{\mathrm{i}}{(4 \pi)^{2}} \frac{r_{\Gamma}}{s t}\left\{\frac { 2 } { \varepsilon _ { I R } ^ { 2 } } \left[\left(\frac{-s-\mathrm{i} \epsilon}{4 \pi \mu^{2}}\right)^{\varepsilon_{I R}}\right.\right. \\
& \left.+\left(\frac{-t-\mathrm{i} \epsilon}{4 \pi \mu^{2}}\right)^{\varepsilon_{I R}}\right]+2 \operatorname{Li}_{2}\left[1-(s+\mathrm{i} \epsilon) f^{0 m}\right] \\
& \left.+2 \operatorname{Li}_{2}\left[1-(t+\mathrm{i} \epsilon) f^{0 m}\right]-2 \frac{\pi^{2}}{3}\right\}+\mathcal{O}\left(\epsilon_{I R}\right) .
\end{aligned}
$$

The above expressions for the one-loop IR divergent scalar box integrals $I_{4}^{K}\left(s, t ;\left\{m_{i}^{2}\right\}\right)$ constitute the main result of this paper. It is important to emphasize that, owing to the fact that we have kept the "causal" i $\epsilon$ systematically throughout the calculation, these expressions are valid for arbitrary values of the relevant kinematic variables: external masses $m_{i}^{2}(i=2,3,4)$ and the Mandelstam variables $s$ and $t$.

As stated in the Introduction, the integrals $I_{4}^{K}\left(s, t,\left\{m_{i}^{2}\right\}\right)$ have been evaluated in Ref. [12] with the help of the partial differential equation technique. The calculation has been performed in the Euclidean region, where all kinematic variables are negative, i.e.,

$$
s, t<0, m_{2}^{2}, m_{3}^{2}, m_{4}^{2}<0,
$$

and the results thus obtained have been analytically continued to the positive values of the kinematic variables (physical region) by applying the following replacements:

$$
s \rightarrow s+\mathrm{i} \epsilon, \quad t \rightarrow t+\mathrm{i} \epsilon, \quad m_{i}^{2} \rightarrow m_{i}^{2}+\mathrm{i} \epsilon .
$$

In order to facilitate the comparison of our results with those of Ref. [12], we have written our results in the same form in which they have been presented in Ref. 12]. A glance at the expressions $69-73)$ reveals that they all have the same general form, namely,

$$
\begin{aligned}
I_{4}^{K}\left(s, t ;\left\{m_{i}^{2}\right\}\right)= & \frac{\mathrm{i}}{(4 \pi)^{2}} r_{\Gamma} g^{K}\left[\frac{G^{K}\left(s, t ; \varepsilon_{I R} ;\left\{m_{i}^{2}\right\}\right)}{\varepsilon_{I R}^{2}}\right. \\
& \left.+H^{K}\left(s, t ;\left\{m_{i}^{2}\right\}\right)\right]+\mathcal{O}\left(\epsilon_{I R}\right) \\
& K \in\{3 m, 2 m h, 2 m e, 1 m, 0 m\} .
\end{aligned}
$$

The IR divergences (both soft and collinear) of the integrals are contained in the first term within the square brackets, while the second term is finite. The function $G^{K}\left(s, t ; \varepsilon_{I R} ;\left\{m_{i}^{2}\right\}\right)$ is represented by a sum of powerlike terms, it depends on $\varepsilon_{I R}$ and is finite in the $\varepsilon_{I R} \rightarrow 0$ limit. As for the function $H^{K}\left(s, t ;\left\{m_{i}^{2}\right\}\right)$, it is given in terms of dilogarithmic functions and constants.

Comparing our results with the corresponding ones of Ref. [12], we find that the expressions for $G^{K}\left(s, t ; \epsilon_{I R} ;\left\{m_{i}^{2}\right\}\right)$ are in agreement. On the other hand, the corresponding expressions for the terms $H^{K}\left(s, t ;\left\{m_{i}^{2}\right\}\right)$ are of different form. Proving the equivalence of our results for the integrals $I_{4}^{K}\left(s, t ;\left\{m_{i}^{2}\right\}\right)$ with those of Ref. [12] then amounts to showing that the expressions for the terms $H^{K}\left(s, t ;\left\{m_{i}^{2}\right\}\right)$ agree numerically. By doing this, we have arrived at the following conclusions: First, the results are in complete agreement in the Euclidean region. Second, for the integrals $I_{4}^{2 m h}\left(s, t ; m_{3}^{2}, m_{4}^{2}\right), I_{4}^{1 m}\left(s, t ; m_{4}^{2}\right)$, and $I_{4}^{0 m}(s, t)$, we have found agreement for arbitrary values of the kinematic variables. Third, the results for the integrals $I_{4}^{3 m}\left(s, t ;, m_{2}^{2}, m_{3}^{2}, m_{4}^{2}\right)$ and $I_{4}^{2 m e}\left(s, t ; m_{2}^{2}, m_{4}^{2}\right)$ do not agree outside the Euclidean region.

The reason for this disagreement is that the analytical continuation from the Euclidean to the physical region as given by Eq. (75) is not well defined for all terms appearing in the expressions for the integrals $I_{4}^{K}\left(s, t ; m_{i}^{2}\right)$ of Ref. [12. This has been pointed out in Ref. [6]. Thus, applying the replacements (75), no cut will be hit by the powerlike terms, logarithms, and the dilogarithms with a single ratio of the kinematical variables. In addition to this kind of terms, the expressions for the integrals $I_{4}^{3 m}\left(s, t ;, m_{2}^{2}, m_{3}^{2}, m_{4}^{2}\right)$ and $I_{4}^{2 m e}\left(s, t ; m_{2}^{2}, m_{4}^{2}\right)$, given in Ref. [12], contain terms of the form

$$
\mathrm{Li}_{2}\left(1-\frac{m_{2}^{2} m_{4}^{2}}{s t}\right)
$$

i.e., the dilogarithms of a product of ratios of the kinematic variables. This type of term requires special care. In order to avoid crossing a cut, in this case one has to make the following replacements:

$$
\begin{aligned}
& \mathrm{Li}_{2}\left(1-\frac{m_{2}^{2} m_{4}^{2}}{s t}\right) \rightarrow \mathrm{Li}_{2}\left(1-\frac{m_{2}^{2}+\mathrm{i} \epsilon}{s+\mathrm{i} \epsilon} \frac{m_{4}^{2}+\mathrm{i} \epsilon}{t+\mathrm{i} \epsilon}\right) \\
& \quad+\eta\left(\frac{m_{2}^{2}+\mathrm{i} \epsilon}{s+\mathrm{i} \epsilon}, \frac{m_{4}^{2}+\mathrm{i} \epsilon}{t+\mathrm{i} \epsilon}\right) \ln \left(1-\frac{m_{2}^{2}+\mathrm{i} \epsilon}{s+\mathrm{i} \epsilon} \frac{m_{4}^{2}+\mathrm{i} \epsilon}{t+\mathrm{i} \epsilon}\right),
\end{aligned}
$$

where the function $\eta$ is defined as

$$
\eta(x, y)=\ln (x y)-\ln (x)-\ln (y),
$$

and arises from the possibility that $x, y$, and $x y$ are not on the same Riemann sheet. Choosing the principal value of the logarithm such that the cut lies along the negative real axis, Eq. (79) can be written as follows:

$$
\begin{aligned}
\eta(x, y)= & 2 \pi \mathrm{i}\{\theta(-\operatorname{Im} x) \theta(-\operatorname{Im} y) \theta(\operatorname{Im} x y) \\
& -\theta(\operatorname{Im} x) \theta(\operatorname{Im} y) \theta(-\operatorname{Im} x y)\} .
\end{aligned}
$$

If the terms of the form given in (77) are analytically continued in accordance with $(78)$, we find that the results of Ref. 12 for the integrals $I_{4}^{3 m}\left(s, t ; m_{2}^{2}, m_{3}^{2}, m_{4}^{2}\right)$ and $I_{4}^{2 m e}\left(s, t ; m_{2}^{2}, m_{4}^{2}\right)$ are numerically equivalent to the corresponding results obtained in this paper for arbitrary values of kinematic variables.

Having thus numerically established the equivalence of the two sets of results for the integrals $I_{4}^{K}\left(s, t ;\left\{m_{i}^{2}\right\}\right)$, our next task is to demonstrate this equivalence analytically, i.e., explicitly. There are, in principle, two approaches to accomplish this. The first approach consists in applying a series of the Hill identities 14] (relating the dilogarithms of different arguments) to the final expression for the integral $Q^{3 m}$ given by Eq. (64), with the aim to express it in terms of the dilogarithms occuring in the final expression for the integral $I_{4}^{3 m}\left(s, t ;\left\{m_{i}^{2}\right\}\right)$ in Ref. [12]. However, because of 
the presence of $i \epsilon$ in the arguments of the dilogarithms in Eq. 64), this turns out to be extremely messy. In the second approach, one tries to achieve the same by recalculating the integral in (61) with an appropriate change of the integration variable. After a lengthy calculation, details of which are presented in Appendix A, we have been able to show that

$$
\begin{aligned}
& Q^{3 m}=-\mathrm{Li}_{2}\left(1-\frac{m_{2}^{2}+\mathrm{i} \epsilon}{s+\mathrm{i} \epsilon}\right)-\mathrm{Li}_{2}\left(1-\frac{m_{2}^{2}+\mathrm{i} \epsilon}{t+\mathrm{i} \epsilon}\right) \\
& -\mathrm{Li}_{2}\left(1-\frac{m_{4}^{2}+\mathrm{i} \epsilon}{s+\mathrm{i} \epsilon}\right)-\mathrm{Li}_{2}\left(1-\frac{m_{4}^{2}+\mathrm{i} \epsilon}{t+\mathrm{i} \epsilon}\right) \\
& +\mathrm{Li}_{2}\left(1-\frac{m_{4}^{2}+\mathrm{i} \epsilon}{s+\mathrm{i} \epsilon} \frac{m_{2}^{2}+\mathrm{i} \epsilon}{t+\mathrm{i} \epsilon}\right)-\frac{1}{2} \ln ^{2}\left(\frac{s+\mathrm{i} \epsilon}{t+\mathrm{i} \epsilon}\right) \\
& +\eta\left(\frac{m_{4}^{2}+\mathrm{i} \epsilon}{s+\mathrm{i} \epsilon}, \frac{m_{2}^{2}+\mathrm{i} \epsilon}{t+\mathrm{i} \epsilon}\right) \ln \left(1-\frac{m_{4}^{2}+\mathrm{i} \epsilon}{s+\mathrm{i} \epsilon} \frac{m_{2}^{2}+\mathrm{i} \epsilon}{t+\mathrm{i} \epsilon}\right) \\
& +\mathcal{O}\left(\varepsilon_{I R}\right) .
\end{aligned}
$$

Although different in form, one can readily prove that this expression for $Q^{3 m}$ is numerically equivalent to that given in Eq. (64) for arbitrary values of kinematic variables. All other integrals $Q^{K}, K \in\{2 m h, 2 m e, 1 m, 0 m\}$ can be derived by taking appropriate zero-mass limits. Combining the expressions thus obtained for $Q^{K}$ with expressions (51), (52), and (54) for $P^{K}$, we arrive at the final expressions for the integrals $I_{4}^{K}\left(s, t ;\left\{m_{i}^{2}\right\}\right)$ which are in agreement with those of Ref. [12] if provided the latter are correctly analytically continued outside the Euclidean region.

\section{Conclusion}

Using the Feynman parameter method, we have calculated in an elegant manner a set of one-loop box scalar integrals with massless internal lines, but containing 0,1 , 2 , or 3 nonzero external masses. To treat IR divergences (both soft and collinear), the dimensional regularization method has been employed. We have kept the causal i $\epsilon$ systematically throughout the calculation. Consequently, the results for these integrals, which appear in the process of evaluating one-loop $(N \geq 5)$-point integrals and in subdiagrams in QCD loop calculations, have been obtained for arbitrary values of the kinematic variables and represent the extension of the results of Ref. [12] outside the Euclidean region.

This work was supported by the Ministry of Science and Technology of the Republic of Croatia under Contract No. 00980102.

\section{Appendix A}

In this Appendix we analytically demonstrate that for Euclidean kinematics our results for the intgerals $I_{4}^{K}\left(s, t ;\left\{m_{i}^{2}\right\}\right)$ are in agreement with the corresponding results obtained in Ref. [12]. As a byproduct, we prove that the correct analytical continuation for the terms of the form given in (77), which appear in the expressions for the integrals $I_{4}^{3 m}\left(s, t ; m_{2}^{2}, m_{3}^{2}, m_{4}^{2}\right)$ and $I_{4}^{2 m e}\left(s, t ; m_{2}^{2}, m_{4}^{2}\right)$ of Ref. [12], is given by Eq. (78).

To accomplish that, we return to the integral $Q^{3 m}$ given in Eq. (61), in which, for convenience, we replace the integration variable $z$ by $y$. Passing to the new integration variable given by

$$
z=1-\frac{y\left(t-m_{2}^{2}\right)-t-\mathrm{i} \epsilon}{y\left(m_{4}^{2}-s\right)-m_{4}^{2}-\mathrm{i} \epsilon},
$$

the integral $Q^{3 m}$ becomes a line integral

$$
\begin{aligned}
Q^{3 m}= & \int_{z_{1}}^{z_{2}} \frac{\mathrm{d} z}{z} \frac{s+t-m_{2}^{2}-m_{4}^{2}}{z\left(m_{4}^{2}-s\right)+s+t-m_{2}^{2}-m_{4}^{2}} \ln (1-z) \\
& +\mathcal{O}\left(\varepsilon_{I R}\right),
\end{aligned}
$$

with the path of integration followed from $z_{1}$ to $z_{2}$, where

$$
z_{1}=1-\frac{t+\mathrm{i} \epsilon}{m_{4}^{2}+\mathrm{i} \epsilon}, \quad z_{2}=1-\frac{m_{2}^{2}+\mathrm{i} \epsilon}{s+\mathrm{i} \epsilon} .
$$

Applying the partial fraction decomposition (23), this integral can be represented as

$$
Q^{3 m}=I+J+\mathcal{O}\left(\varepsilon_{I R}\right)
$$

where

$$
I=\int_{z_{1}}^{z_{2}} \frac{\mathrm{d} z}{z} \ln (1-z)
$$

and

$$
J=-\int_{z_{1}}^{z_{2}} \mathrm{~d} z \frac{m_{4}^{2}-s}{z\left(m_{4}^{2}-s\right)+s+t-m_{2}^{2}-m_{4}^{2}} \ln (1-z) .
$$

Let us now consider these two integrals in turn.

The integrand in (86) has a first-order pole at $z=0$ and the logarithmic branch cut extending from 1 to $\infty$. It follows from Eq. (84) that, depending on the values of the parameters $s, t, m_{2}^{2}, m_{4}^{2}$, it might happen that the line connecting the points $z_{1}$ and $z_{2}$ crosses the real axis. In this case, as it can be seen from Eq. (82), the crossing occurs at the point $z=0$ - the pole of the integrand. Observe, however, that the residue of the integrand at $z=0$ is zero. A consequence of this is that, regardless of whether the line connecting $z_{1}$ and $z_{2}$ crosses the real axis or not, we are allowed to assume that it passes through the point $z=0$. Consequently, the line between $z_{1}$ and $z_{2}$ can be decomposed into two segments: one connecting $z_{1}$ and 0 , and the other connecting 0 and $z_{2}$. In view of this, the integral $I$ can be rewritten in the form

$$
I=\int_{0}^{z_{2}} \frac{\mathrm{d} z}{z} \ln (1-z)-\int_{0}^{z_{1}} \frac{\mathrm{d} z}{z} \ln (1-z)
$$

By changing the integration variables $z \rightarrow z z_{2}$ and $z \rightarrow$ $z z_{1}$ in the first and second integral, respectively, and taking formula (47) into account, we obtain the result

$$
I=-\mathrm{Li}_{2}\left(1-\frac{m_{2}^{2}+\mathrm{i} \epsilon}{s+\mathrm{i} \epsilon}\right)+\mathrm{Li}_{2}\left(1-\frac{t+\mathrm{i} \epsilon}{m_{4}^{2}+\mathrm{i} \epsilon}\right) .
$$


Applying the transformation

$$
\mathrm{Li}_{2}\left(1-\frac{1}{y}\right)=-\mathrm{Li}_{2}(1-y)-\frac{1}{2} \ln ^{2} y
$$

to the second term on the right-hand side in (89), the final expression for the integral $I$ is found to be

$$
\begin{aligned}
I= & -\mathrm{Li}_{2}\left(1-\frac{m_{2}^{2}+\mathrm{i} \epsilon}{s+\mathrm{i} \epsilon}\right)-\mathrm{Li}_{2}\left(1-\frac{m_{4}^{2}+\mathrm{i} \epsilon}{t+\mathrm{i} \epsilon}\right) \\
& -\frac{1}{2} \ln ^{2}\left(\frac{m_{4}^{2}+\mathrm{i} \epsilon}{t+\mathrm{i} \epsilon}\right) .
\end{aligned}
$$

Turning now to the integral $J$ in (87), we switch back to the old integration variable as a result of which the integral takes the form

$$
J=J_{1}+J_{2}+J_{3},
$$

where

$$
\begin{aligned}
J_{1}= & -\int_{0}^{1} \mathrm{~d} y \frac{m_{4}^{2}-s}{y\left(m_{4}^{2}-s\right)-m_{4}^{2}-\mathrm{i} \epsilon} \\
& \times \ln \left[y\left(m_{4}^{2}-s\right)-m_{4}^{2}-\mathrm{i} \epsilon\right] \\
J_{2}= & \int_{0}^{1} \mathrm{~d} y \frac{m_{4}^{2}-s}{y\left(m_{4}^{2}-s\right)-m_{4}^{2}-\mathrm{i} \epsilon} \ln (-t-\mathrm{i} \epsilon),
\end{aligned}
$$

and

$$
J_{3}=\int_{0}^{1} \mathrm{~d} y \frac{m_{4}^{2}-s}{y\left(m_{4}^{2}-s\right)-m_{4}^{2}-\mathrm{i} \epsilon} \ln \left(1-y \frac{t-m_{2}^{2}}{t+\mathrm{i} \epsilon}\right) .
$$

The integrals $J_{1}$ and $J_{2}$ are elementary, and are readily evaluated. The results are

$$
\begin{aligned}
& J_{1}=-\frac{1}{2}\left[\ln ^{2}(-s-\mathrm{i} \epsilon)-\ln ^{2}\left(-m_{4}^{2}-\mathrm{i} \epsilon\right)\right], \\
& J_{2}=\ln (-t-\mathrm{i} \epsilon) \ln \left(\frac{s+\mathrm{i} \epsilon}{m_{4}^{2}+\mathrm{i} \epsilon}\right) .
\end{aligned}
$$

In order to evaluate the integral $J_{3}$, we proceed by adding and subtracting the following expression:

$$
\begin{gathered}
\int_{0}^{1} \frac{\mathrm{d} y}{y} \ln \left(1-y \frac{t-m_{2}^{2}}{t+\mathrm{i} \epsilon}\right)+\int_{0}^{1} \frac{\mathrm{d} y}{y} \ln (1-y) \\
+\int_{0}^{1} \mathrm{~d} y \frac{1}{y-\frac{m_{4}^{2}+\mathrm{i} \epsilon}{m_{4}^{2}-s}} \ln (1-y) .
\end{gathered}
$$

After a simple algebraic reduction, the integral $J_{3}$ can be represented in the form

$$
J_{3}=J_{3,1}+J_{3,2},
$$

where

$$
\begin{aligned}
J_{3,1} & =\int_{0}^{1} \frac{\mathrm{d} y}{y} \ln \left(1-y \frac{t-m_{2}^{2}}{t+\mathrm{i} \epsilon}\right)-\int_{0}^{1} \frac{\mathrm{d} y}{y} \ln (1-y) \\
& +\int_{0}^{1} \mathrm{~d} y \frac{1}{y-\frac{m_{4}^{2}+\mathrm{i} \epsilon}{m_{4}^{2}-s}} \ln (1-y) \\
= & -\mathrm{Li}_{2}\left(1-\frac{m_{2}^{2}+\mathrm{i} \epsilon}{t+\mathrm{i} \epsilon}\right)+\frac{\pi^{2}}{6}-\mathrm{Li}_{2}\left(1-\frac{m_{4}^{2}+\mathrm{i} \epsilon}{s+\mathrm{i} \epsilon}\right)
\end{aligned}
$$

and

$$
J_{3,2}=-\int_{0}^{1} \mathrm{~d} y\left[\frac{1}{y-\frac{m_{4}^{2}+\mathrm{i} \epsilon}{m_{4}^{2}-s}}-\frac{1}{y}\right] \ln \left(\frac{1-y}{1-y \frac{t-m_{2}^{2}}{t+\mathrm{i} \epsilon}}\right) .
$$

Next, consider the integral $J_{3,2}$. Introducing a new integration variable

$$
z=\frac{1-y}{1-y \frac{t-m_{2}^{2}}{t+\mathrm{i} \epsilon}}
$$

and the notation

$$
a=\frac{\left(m_{2}^{2}+\mathrm{i} \epsilon\right)\left(m_{4}^{2}+\mathrm{i} \epsilon\right)}{(s+\mathrm{i} \epsilon)(t+\mathrm{i} \epsilon)},
$$

the integral can be cast into the form

$$
J_{3,2}=\int_{0}^{1} \frac{\mathrm{d} z}{z} \ln (1-z)+\int_{0}^{1} \mathrm{~d} z \frac{1}{z-\frac{1}{1-a}} \ln z .
$$

Notice that, in accordance with (101), the integral $J_{3,2}$ is given by Eq. (103) as a line integral in the complex $z$-plane with the integration path having only the end-points on the real axis at $z=0$ and $z=1$. Adding and subtracting the integral of the form

$$
\int_{0}^{1} \mathrm{~d} z \frac{1}{z-\frac{1}{1-a}} \ln (1-a)
$$

in the second integral in $(103)$ allows us to write the integral $J_{3,2}$ as

$$
\begin{aligned}
J_{3,2}= & \int_{0}^{1} \frac{\mathrm{d} z}{z} \ln (1-z)+\int_{0}^{1} \mathrm{~d} z \frac{1}{z-\frac{1}{1-a}}[\ln z+\ln (1-a)] \\
& -\int_{0}^{1} \mathrm{~d} z \frac{1}{z-\frac{1}{1-a}} \ln (1-a) .
\end{aligned}
$$

It should be observed that the residue of the first integral on the right-hand side in (101) at the pole $z=0$ is equal to zero. The same is true for the second integral at the pole $z=1 /(1-a)$. Therefore, the integration path in both of these integrals can be taken to follow the real axis from 0 to 1 . After evaluating the first two integrals in (104) and passing to the old integration variable in the third integral, we arrive at the following expression for $J_{3,2}$ :

$$
\begin{aligned}
& J_{3,2}=-\frac{\pi^{2}}{6}+\mathrm{Li}_{2}(1-a)+\ln (a) \ln (1-a)+\ln (1-a) \\
& \times \int_{0}^{1} \mathrm{~d} y \frac{s t-m_{2}^{2} m_{4}^{2}+\mathrm{i} \epsilon\left(s+t-m_{2}^{2}-m_{4}^{2}\right)}{\left[y\left(m_{4}^{2}-s\right)-m_{4}^{2}-\mathrm{i} \epsilon\right]\left[y\left(t-m_{2}^{2}\right)-t-\mathrm{i} \epsilon\right]}(105)
\end{aligned}
$$


Carrying out the remaining integration, we obtain the final expression

$J_{3,2}=-\frac{\pi^{2}}{6}+\operatorname{Li}_{2}(1-a)+\eta\left(\frac{m_{4}^{2}+\mathrm{i} \epsilon}{s+\mathrm{i} \epsilon}, \frac{m_{2}^{2}+\mathrm{i} \epsilon}{t+\mathrm{i} \epsilon}\right) \ln (1-a)$,

where the function $\eta(x, y)$ is defined by Eq. (79). Now, substituting $(99)$ and $(106)$ into $(98)$, we find the integral $J_{3}$ to be given by

$$
\begin{aligned}
J_{3}= & -\mathrm{Li}_{2}\left(1-\frac{m_{2}^{2}+\mathrm{i} \epsilon}{t+\mathrm{i} \epsilon}\right)-\mathrm{Li}_{2}\left(1-\frac{m_{4}^{2}+\mathrm{i} \epsilon}{s+\mathrm{i} \epsilon}\right) \\
& +\mathrm{Li}_{2}\left(1-\frac{m_{4}^{2}+\mathrm{i} \epsilon}{s+\mathrm{i} \epsilon} \frac{m_{2}^{2}+\mathrm{i} \epsilon}{t+\mathrm{i} \epsilon}\right) \\
& +\eta\left(\frac{m_{4}^{2}+\mathrm{i} \epsilon}{s+\mathrm{i} \epsilon}, \frac{m_{2}^{2}+\mathrm{i} \epsilon}{t+\mathrm{i} \epsilon}\right) \ln \left(1-\frac{m_{4}^{2}+\mathrm{i} \epsilon}{s+\mathrm{i} \epsilon} \frac{m_{2}^{2}+\mathrm{i} \epsilon}{t+\mathrm{i} \epsilon}\right) .
\end{aligned}
$$

Finally, having obtained all the necessary ingredients, we now combine them to obtain (81), which is the desired result.

\section{Appendix B}

By expanding the powerlike terms appearing in Eqs. (69)(73), and the prefactor $r_{\Gamma}$ defined by (68), we find that the integrals under consideration can be written in the generic form

$$
\begin{aligned}
I_{4}^{K}= & \frac{\mathrm{i}}{(4 \pi)^{2}} g^{K}\left(\frac{A^{K}}{\varepsilon_{I R}^{2}}+\frac{B^{K}}{\varepsilon_{I R}}+C_{1}^{K}+C_{2}^{K}\right)+\mathcal{O}\left(\varepsilon_{I R}\right), \\
& K \in\{3 m, 2 m h, 2 m e, 1 m, 0 m\}
\end{aligned}
$$

The functions $g^{K}$ appearing above are defined by (29). For convenience, the finite parts have been decomposed into two terms where the term $C_{1}^{K}$ originates from the expansion of the product of the $r_{\Gamma}$ prefactor with the powerlike terms.

The double-pole parts $A^{K}$, the single-pole parts $B^{K}$, as well as the finite parts $C_{1}^{K}$ and $C_{2}^{K}$ of the individual integrals are listed below.

$$
\begin{aligned}
A^{3 m} & =0 \\
B^{3 m} & =\ln \left(\frac{s+\mathrm{i} \epsilon}{m_{2}^{2}+\mathrm{i} \epsilon}\right)+\ln \left(\frac{t+\mathrm{i} \epsilon}{m_{4}^{2}+\mathrm{i} \epsilon}\right) \\
C_{1}^{3 m} & =\left[\gamma_{E}+\ln \left(\frac{-s-\mathrm{i} \epsilon}{4 \pi \mu^{2}}\right)\right]^{2}+\left[\gamma_{E}+\ln \left(\frac{-t-\mathrm{i} \epsilon}{4 \pi \mu^{2}}\right)\right]^{2} \\
& -\left[\gamma_{E}+\ln \left(\frac{-m_{2}^{2}-\mathrm{i} \epsilon}{4 \pi \mu^{2}}\right)\right]^{2}-\left[\gamma_{E}+\ln \left(\frac{-m_{3}^{2}-\mathrm{i} \epsilon}{4 \pi \mu^{2}}\right)\right]^{2} \\
& -\left[\gamma_{E}+\ln \left(\frac{-m_{4}^{2}-\mathrm{i} \epsilon}{4 \pi \mu^{2}}\right)\right]^{2} \\
& +\frac{1}{2}\left[\gamma_{E}+\ln \left(\frac{-m_{3}^{2}-\mathrm{i} \epsilon}{4 \pi \mu^{2}}\right)+\ln \left(\frac{m_{4}^{2}+\mathrm{i} \epsilon}{s+\mathrm{i} \epsilon}\right)\right]^{2} \\
& +\frac{1}{2}\left[\gamma_{E}+\ln \left(\frac{-m_{3}^{2}-\mathrm{i} \epsilon}{4 \pi \mu^{2}}\right)+\ln \left(\frac{m_{2}^{2}+\mathrm{i} \epsilon}{t+\mathrm{i} \epsilon}\right)\right]^{2},
\end{aligned}
$$

$$
\begin{aligned}
& C_{2}^{3 m}=2 \mathrm{Li}_{2}\left(1-\frac{m_{2}^{2}+\mathrm{i} \epsilon}{t+\mathrm{i} \epsilon}\right)+2 \mathrm{Li}_{2}\left(1-\frac{m_{4}^{2}+\mathrm{i} \epsilon}{s+\mathrm{i} \epsilon}\right) \\
& +2 \mathrm{Li}_{2}\left[1-(s+\mathrm{i} \epsilon) f^{3 m}\right]+2 \operatorname{Li}_{2}\left[1-(t+\mathrm{i} \epsilon) f^{3 m}\right] \\
& -2 \mathrm{Li}_{2}\left[1-\left(m_{2}^{2}+\mathrm{i} \epsilon\right) f^{3 m}\right]-2 \mathrm{Li}_{2}\left[1-\left(m_{4}^{2}+\mathrm{i} \epsilon\right) f^{3 m}\right] .
\end{aligned}
$$

$$
\begin{aligned}
& A^{2 m h}=1 \\
& B^{2 m h}=\gamma_{E}+\ln \left(\frac{-t-\mathrm{i} \epsilon}{4 \pi \mu^{2}}\right)+\ln \left(\frac{s+\mathrm{i} \epsilon}{m_{4}^{2}+\mathrm{i} \epsilon}\right) \\
&+\ln \left(\frac{t+\mathrm{i} \epsilon}{m_{3}^{2}+\mathrm{i} \epsilon}\right), \\
& C_{1}^{2 m h}=\left[\gamma_{E}+\ln \left(\frac{-s-\mathrm{i} \epsilon}{4 \pi \mu^{2}}\right)\right]^{2}+\left[\gamma_{E}+\ln \left(\frac{-t-\mathrm{i} \epsilon}{4 \pi \mu^{2}}\right)\right]^{2} \\
&-\left[\gamma_{E}+\ln \left(\frac{-m_{3}^{2}-\mathrm{i} \epsilon}{4 \pi \mu^{2}}\right)\right]^{2}-\left[\gamma_{E}+\ln \left(\frac{-m_{4}^{2}-\mathrm{i} \epsilon}{4 \pi \mu^{2}}\right)\right]^{2} \\
&+\frac{1}{2}\left[\gamma_{E}+\ln \left(\frac{-m_{3}^{2}-\mathrm{i} \epsilon}{4 \pi \mu^{2}}\right)+\ln \left(\frac{m_{4}^{2}+\mathrm{i} \epsilon}{s+\mathrm{i} \epsilon}\right)\right]^{2}-\frac{\pi^{2}}{12}, \\
& C_{2}^{2 m h}=2 \mathrm{Li}_{2}\left(1-\frac{m_{4}^{2}+\mathrm{i} \epsilon}{s+\mathrm{i} \epsilon}\right)-2 \mathrm{Li}_{2}\left(1-\frac{m_{3}^{2}+\mathrm{i} \epsilon}{t+\mathrm{i} \epsilon}\right) \\
&+2 \mathrm{Li}_{2}\left[1-(s+\mathrm{i} \epsilon) f^{2 m h}\right]+2 \operatorname{Li}_{2}\left[1-(t+\mathrm{i} \epsilon) f^{2 m h}\right] \\
&-2 \operatorname{Li}_{2}\left[1-\left(m_{4}^{2}+\mathrm{i} \epsilon\right) f^{2 m h}\right] .
\end{aligned}
$$

$$
\begin{aligned}
& A^{2 m e}=0 \text {, } \\
& B^{2 m e}=2 \ln \left(\frac{s+\mathrm{i} \epsilon}{m_{2}^{2}+\mathrm{i} \epsilon}\right)+2 \ln \left(\frac{t+\mathrm{i} \epsilon}{m_{4}^{2}+\mathrm{i} \epsilon}\right), \\
& C_{1}^{2 m e}=\left[\gamma_{E}+\ln \left(\frac{-s-\mathrm{i} \epsilon}{4 \pi \mu^{2}}\right)\right]^{2}+\left[\gamma_{E}+\ln \left(\frac{-t-\mathrm{i} \epsilon}{4 \pi \mu^{2}}\right)\right]^{2} \\
& -\left[\gamma_{E}+\ln \left(\frac{-m_{2}^{2}-\mathrm{i} \epsilon}{4 \pi \mu^{2}}\right)\right]^{2}-\left[\gamma_{E}+\ln \left(\frac{-m_{4}^{2}-\mathrm{i} \epsilon}{4 \pi \mu^{2}}\right)\right]^{2}, \\
& C_{2}^{2 m e}=2 \operatorname{Li}_{2}\left[1-(s+\mathrm{i} \epsilon) f^{2 m e}\right]+2 \operatorname{Li}_{2}\left[1-(t+\mathrm{i} \epsilon) f^{2 m e}\right] \\
& -2 \mathrm{Li}_{2}\left[1-\left(m_{2}^{2}+\mathrm{i} \epsilon\right) f^{2 m e}\right]-2 \operatorname{Li}_{2}\left[1-\left(m_{4}^{2}+\mathrm{i} \epsilon\right) f^{2 m e}\right] . \\
& A^{1 m}=2 \text {, } \\
& B^{1 m}=2 \gamma_{E}+2 \ln \left(\frac{-s-\mathrm{i} \epsilon}{4 \pi \mu^{2}}\right)+2 \ln \left(\frac{-t-\mathrm{i} \epsilon}{4 \pi \mu^{2}}\right) \\
& -2 \ln \left(\frac{-m_{4}^{2}-\mathrm{i} \epsilon}{4 \pi \mu^{2}}\right) \text {, } \\
& C_{1}^{1 m}=\left[\gamma_{E}+\ln \left(\frac{-s-\mathrm{i} \epsilon}{4 \pi \mu^{2}}\right)\right]^{2}+\left[\gamma_{E}+\ln \left(\frac{-t-\mathrm{i} \epsilon}{4 \pi \mu^{2}}\right)\right]^{2} \\
& -\left[\gamma_{E}+\ln \left(\frac{-m_{4}^{2}-\mathrm{i} \epsilon}{4 \pi \mu^{2}}\right)\right]^{2}-\frac{\pi^{2}}{6}, \\
& C_{2}^{1 m}=2 \operatorname{Li}_{2}\left[1-(s+\mathrm{i} \epsilon) f^{1 m}\right]+2 \operatorname{Li}_{2}\left[1-(t+\mathrm{i} \epsilon) f^{1 m}\right]
\end{aligned}
$$




$$
-2 \mathrm{Li}_{2}\left[1-\left(m_{4}^{2}+\mathrm{i} \epsilon\right) f^{1 m}\right]-\frac{\pi^{2}}{3}
$$

$$
\begin{aligned}
A^{0 m} & =4, \\
B^{0 m} & =4 \gamma_{E}+2 \ln \left(\frac{-s-\mathrm{i} \epsilon}{4 \pi \mu^{2}}\right)+2 \ln \left(\frac{-t-\mathrm{i} \epsilon}{4 \pi \mu^{2}}\right), \\
C_{1}^{0 m} & =\left[\gamma_{E}+\ln \left(\frac{-s-\mathrm{i} \epsilon}{4 \pi \mu^{2}}\right)\right]^{2}+\left[\gamma_{E}+\ln \left(\frac{-t-\mathrm{i} \epsilon}{4 \pi \mu^{2}}\right)\right]^{2}-\frac{\pi^{2}}{3}, \\
C_{2}^{0 m} & =2 \operatorname{Li}_{2}\left[1-(s+\mathrm{i} \epsilon) f^{0 m}\right]+2 \operatorname{Li}_{2}\left[1-(t+\mathrm{i} \epsilon) f^{0 m}\right] \\
& -2 \frac{\pi^{2}}{3} .
\end{aligned}
$$

In the above expressions, $\gamma_{E}=0.5722$ is the Euler constant.

The pole and finite parts for the integrals $I_{4}^{K}\left(s, t ; m_{i}^{2}\right)$ obtained in Ref. 12, but with the correct analytical continuation outside the Euclidean region for the integrals $I_{4}^{3 m}\left(s, t ; m_{2}^{2}, m_{3}^{2}, m_{4}^{2}\right)$ and $I_{4}^{2 m e}\left(s, t ; m_{2}^{2}, m_{4}^{2}\right)$, can be obtained simply by replacing the terms $C_{2}^{K}\left(s, t ; m_{i}^{2}\right)$ given above by the corresponding values listed below:

$$
\begin{aligned}
& C_{2}^{3 m}=-\ln ^{2}\left(\frac{s+\mathrm{i} \epsilon}{t+\mathrm{i} \epsilon}\right) \\
& -2 \mathrm{Li}_{2}\left(1-\frac{m_{2}^{2}+\mathrm{i} \epsilon}{s+\mathrm{i} \epsilon}\right)-2 \mathrm{Li}_{2}\left(1-\frac{m_{4}^{2}+\mathrm{i} \epsilon}{t+\mathrm{i} \epsilon}\right) \\
& +2 \mathrm{Li}_{2}\left(1-\frac{m_{2}^{2}+\mathrm{i} \epsilon}{s+\mathrm{i} \epsilon} \frac{m_{4}^{2}+\mathrm{i} \epsilon}{t+\mathrm{i} \epsilon}\right) \\
& +2 \eta\left(\frac{m_{2}^{2}+\mathrm{i} \epsilon}{s+\mathrm{i} \epsilon}, \frac{m_{4}^{2}+\mathrm{i} \epsilon}{t+\mathrm{i} \epsilon}\right) \ln \left(1-\frac{m_{2}^{2}+\mathrm{i} \epsilon}{s+\mathrm{i} \epsilon} \frac{m_{4}^{2}+\mathrm{i} \epsilon}{t+\mathrm{i} \epsilon}\right), \\
& C_{2}^{2 m h}=-\ln ^{2}\left(\frac{s+\mathrm{i} \epsilon}{t+\mathrm{i} \epsilon}\right) \\
& -2 \operatorname{Li}_{2}\left(1-\frac{m_{3}^{2}+\mathrm{i} \epsilon}{t+\mathrm{i} \epsilon}\right)-2 \operatorname{Li}_{2}\left(1-\frac{m_{4}^{2}+\mathrm{i} \epsilon}{t+\mathrm{i} \epsilon}\right)
\end{aligned}
$$

$$
\begin{aligned}
& C_{2}^{2 m e}=-\ln ^{2}\left(\frac{s+\mathrm{i} \epsilon}{t+\mathrm{i} \epsilon}\right) \\
& \quad-2 \operatorname{Li}_{2}\left(1-\frac{m_{2}^{2}+\mathrm{i} \epsilon}{s+\mathrm{i} \epsilon}\right)-2 \mathrm{Li}_{2}\left(1-\frac{m_{2}^{2}+\mathrm{i} \epsilon}{t+\mathrm{i} \epsilon}\right) \\
& \quad-2 \operatorname{Li}_{2}\left(1-\frac{m_{4}^{2}+\mathrm{i} \epsilon}{s+\mathrm{i} \epsilon}\right)-2 \mathrm{Li}_{2}\left(1-\frac{m_{4}^{2}+\mathrm{i} \epsilon}{t+\mathrm{i} \epsilon}\right) \\
& +2 \operatorname{Li}_{2}\left(1-\frac{m_{2}^{2}+\mathrm{i} \epsilon}{s+\mathrm{i} \epsilon} \frac{m_{4}^{2}+\mathrm{i} \epsilon}{t+\mathrm{i} \epsilon}\right) \\
& +2 \eta\left(\frac{m_{2}^{2}+\mathrm{i} \epsilon}{s+\mathrm{i} \epsilon}, \frac{m_{4}^{2}+\mathrm{i} \epsilon}{t+\mathrm{i} \epsilon}\right) \ln \left(1-\frac{m_{2}^{2}+\mathrm{i} \epsilon}{s+\mathrm{i} \epsilon} \frac{m_{4}^{2}+\mathrm{i} \epsilon}{t+\mathrm{i} \epsilon}\right),
\end{aligned}
$$

$$
\begin{aligned}
& -2 \operatorname{Li}_{2}\left(1-\frac{m_{4}^{2}+\mathrm{i} \epsilon}{s+\mathrm{i} \epsilon}\right)-2 \operatorname{Li}_{2}\left(1-\frac{m_{4}^{2}+\mathrm{i} \epsilon}{t+\mathrm{i} \epsilon}\right) \\
& C_{2}^{0 m}=-\ln ^{2}\left(\frac{s+\mathrm{i} \epsilon}{t+\mathrm{i} \epsilon}\right)-\pi^{2}
\end{aligned}
$$

As it is readily seen from the above expressions, the integrals $I_{4}^{K}\left(s, t ;\left\{m_{i}^{2}\right\}\right)$ are real in the Euclidean region . Outside of this region, however, the integrals acquire an imaginary part. Being given in terms of logarithms and dilogarithms, their imaginary parts can be easily determined.

Thus, with the usual definition of the logarithms on the branch cut $-\infty<\mathrm{z} \leq 0$, one has

$$
\ln (y \pm i \epsilon)=\ln |y| \pm \mathrm{i} \pi \theta(-y) .
$$

Next, as it is seen from Eq. (47), the function $\operatorname{Li}_{2}(y)$ develops an imaginary part for $y \geq 1$, and

$$
\begin{gathered}
\operatorname{Re} \operatorname{Li}_{2}(y \pm \mathrm{i} \epsilon)=-\mathrm{Li}_{2}\left(\frac{1}{y}\right)-\frac{1}{2} \ln ^{2} y+\frac{\pi^{2}}{3}, \\
\operatorname{Im} \operatorname{Li}_{2}(y \pm \mathrm{i} \epsilon)= \pm \pi \ln y
\end{gathered}
$$

\section{References}

1. M. L. Mangano, S. J. Parke, Phys. Rep. 200, 301 (1991); P. Draggiotis, R. H. P. Kleiss, C. G. Papadopoulos, Phys. Lett. B 439, 157 (1998); F. Caravaglios, M. L. Mangano, M. Moretti, R. Pittau, Nucl. Phys B 539, 215 (1999).

2. G. Passarino, M. Veltman, Nucl. Phys. B 160, 151 (1979); G. J. van Oldenborgh, J. A. M. Vermaseren, Z. Phys. C 46, 425 (1990); W. L. van Neerven, J. A. M. Vermaseren, Phys. Lett. B 137, 241 (1984).

3. A. I. Davydychev, Phys. Lett. B 263, 107 (1991).

4. Z. Bern, L. Dixon, D. A. Kosower, Phys. Lett. B 302, 299 (1993); E. ibid Phys. Lett. B 318, 649 (1993).

5. O. V. Tarasov, Phys. Rev. D 54, 6479 (1996); J. Fleischer, F. Jegerlehner, O. V. Tarasov, Nucl. Phys. B 566, 423 (2000).

6. T. Binoth, J. Ph. Guillet, G. Heinrich, Nucl. Phys. B 572, 361 (2000).

7. J. M. Campbell, E. W. N. Glover, D. J. Miller, Nucl. Phys. B 498, 397 (1997).

8. G.'t Hooft, M. Veltman, Nucl. Phys. B 153, 365 (1979).

9. A. Denner, U. Nierste, R. Scharf, Nucl. Phys. B 367, 637 (1991).

10. K. Fabricius, I. Schmitt, Z. Phys. C 3, 51 (1979).

11. S. Papadopoulos, A. P. Contogouris, J. Ralston, Phys. Rev. D 25, 2218 (1982).

12. Z. Bern, L. Dixon, D. A. Kosower, Nucl. Phys. B 412, 751 (1994).

13. R. Karplus, M. Neuman, Phys. Rev. 83, 776 (1951).

14. L. Lewin, Polylogarithms and associated functions (North Holland, New York 1981).

$$
C_{2}^{1 m}=-\ln ^{2}\left(\frac{s+\mathrm{i} \epsilon}{t+\mathrm{i} \epsilon}\right)-\frac{\pi^{2}}{3}
$$

